Noname manuscript No.

(will be inserted by the editor)

\title{
A stabilized Nitsche overlapping mesh method for the Stokes problem
}

\author{
André Massing • Mats G. Larson • Anders Logg • \\ Marie E. Rognes
}

Received: November 21, 2018/ Accepted:

\begin{abstract}
We develop a Nitsche-based formulation for a general class of stabilized finite element methods for the Stokes problem posed on a pair of overlapping, non-matching meshes. By extending the least-squares stabilization to the overlap region, we prove that the method is stable, consistent, and optimally convergent. To avoid an ill-conditioned linear algebra system, the scheme is augmented by a least-squares term measuring the discontinuity of the solution in the overlap region of the two meshes. As a consequence, we may prove an estimate for the condition number of the resulting stiffness matrix that is independent of the location of the interface. Finally, we present numerical examples in three spatial dimensions illustrating and confirming the theoretical results.
\end{abstract}

Keywords Fictitious domain · Stokes problem · stabilized finite element methods · Nitsche's method

Mathematics Subject Classification (2010) MSC 65N12 · MSC 65N30 - MSC 76D07

\section{Introduction}

Overlapping mesh methods offer many advantages over standard finite element methods that require the generation of a single conforming mesh resolving the full computational domain. With overlapping mesh methods, the computational domain may instead be described by a set of overlapping and non-matching meshes. In particular, different subdomains may be meshed independently and then collected to form the full domain. This feature is particularly useful in engineering applications where meshes for physical components may be reused in different configurations. Another important example is the simulation of the flow around a complex object embedded in a channel. One may then create a mesh that discretizes a fixed and simple domain such as a cube or a sphere surrounding the complex object. This mesh may then be imposed on top of a fixed background mesh for the simulation of the flow around the object inserted at different locations in a domain

André Massing

Simula Research Laboratory, Oslo, Norway

Tel.: +4746957401

Fax: +4767828201

E-mail: massing@simula.no

Mats G. Larson

Department of Mathematics, Umeå University, Umeå, Sweden

Anders Logg

Simula Research Laboratory, Oslo, Norway

Marie E. Rognes

Simula Research Laboratory, Oslo, Norway 


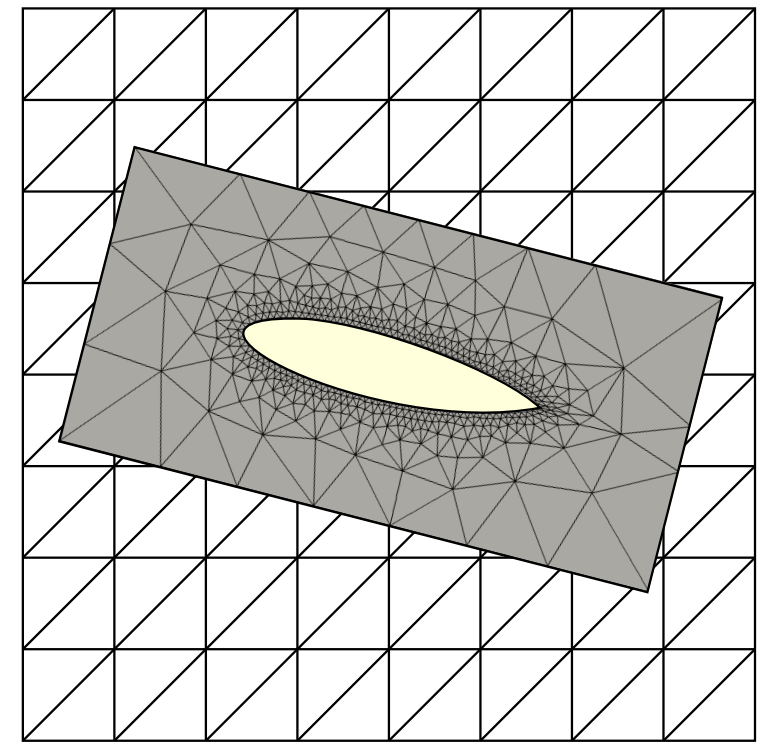

Fig. 1.1 The stabilized Nitsche overlapping mesh method presented in this work allows the simulation of Stokes flow around a complex object (here a simple two-dimensional airfoil) described by a matching mesh of its surroundings imposed on top of a non-matching fixed background mesh.

of interest. A particular advantage of this approach is that it allows the creation of a fixed graded mesh to resolve boundary layers close to the surface of the complex object. This is illustrated in Figure 1.1 for a simple two-dimensional airfoil embedded in a channel.

In this work, we introduce an overlapping mesh method for Stokes flow with constant viscosity across the artificial mesh interface. The Stokes problem reads: find the velocity $\boldsymbol{u}: \Omega \subset \mathbb{R}^{d} \rightarrow \mathbb{R}^{d}$ and the pressure $p: \Omega \rightarrow \mathbb{R}$ such that

$$
\begin{aligned}
-\Delta \boldsymbol{u}+\nabla p=\boldsymbol{f} & \text { in } \Omega, \\
\nabla \cdot \boldsymbol{u}=0 & \text { in } \Omega, \\
\boldsymbol{u}=\boldsymbol{g} & \text { on } \partial \Omega,
\end{aligned}
$$

where $\Omega$ denotes a bounded domain in $\mathbb{R}^{d}, d=2$ or 3 , with Lipschitz boundary $\partial \Omega$, and where $\boldsymbol{f} \in L^{2}(\Omega)$ and $\boldsymbol{g} \in H^{1 / 2}(\partial \Omega)$ are given functions. To satisfy $1.1 \mathrm{~b}$, we assume that the mean value of $\boldsymbol{g} \cdot \boldsymbol{n}$ vanishes; $\boldsymbol{n}$ denoting the outward unit normal to $\partial \Omega$.

Our formulation is based on a general stabilized Galerkin finite element method for the Stokes problem and enforcement of the interface conditions via Nitsche's method. In order to prove stability, we let the least-squares stabilization terms extend to all elements that intersect the computational domain. As a result, these terms will appear twice in any overlap regions. In addition, we include a certain least-squares term penalizing the difference between the velocity solutions in the overlap region. This allows us to prove stability and optimal order error estimates as well as to control the condition number of the resulting algebraic problem.

The method proposed here can be viewed as an extension to the Stokes problem of earlier work by Hansbo et al. [13] who developed a Nitsche overlapping mesh method for a second order elliptic model problem. Also, Becker et al. [5] presented a Nitsche extended finite element method for incompressible elasticity based on low-order $\left(\left[P_{1}^{c}\right]^{d} \times P_{0}\right)$ elements. Moreover, the least-squares penalty of the velocity differences is related to the mesh tying approach proposed by Day and Bochev [7, who formulate a least-squares problem for a system consisting of the partial differential equation together with the interface conditions. Note that in our method, the interface conditions are enforced using Nitsche's method, while the least-squares terms on the overlap are only included 
to prove the stability of the method and to control the condition number. In a related work [23, we present a stabilized Nitsche fictitious domain method for the Stokes problem.

The implementation of the overlapping mesh method in three space dimensions is a challenging problem. A realization of the method proposed in this work entails computing the intersection of arbitrarily superimposed tetrahedral meshes and integration over arbitrarily cut tetrahedra. Such a realization has been developed as part of the $\mathrm{C}++$ library DOLFIN-OLM (http://launchpad. net/dolfin-olm) extending the FEniCS Project software [20, 19, 18, 17, 21, 1, 2]. For a discussion of the computational aspects, we refer to our previous work 22 and the related paper 23 .

The remainder of this work is organized as follows. We first summarize our assumptions and notation in Section 2. The overlapping mesh method is then formulated in Section 3 . Sections 46 are devoted to the stability and a priori error analysis of the proposed method, while the condition number estimate is presented in Section 7. Finally, we demonstrate the proposed method for a sample application in Section 8, and present numerical convergence results and condition number estimates to support our theoretical results.

\section{Preliminaries}

In this section, we review the notation used throughout the remainder of this work. We also summarize a standard stabilized Stokes formulation to lay the foundations for the formulation of the overlapping mesh method in Section 3 .

\subsection{Finite element spaces}

In what follows, $H^{s}(\Omega)$ denotes the standard Sobolev space of order $s \in \mathbb{N}$, defined on an open and bound domain $\Omega$ with Lipschitz boundary $\partial \Omega$. We write $(\cdot, \cdot)_{s, \Omega},\|\cdot\|_{s, \Omega}$ and $|\cdot|_{s, \Omega}$ for the inner product, norm and semi-norm on $H^{s}(\Omega)$, respectively. The index $s$ will be dropped when $s=0$.

For a given, shape-regular tessellation $\mathcal{T}$ of $\Omega$, we let the associated discrete velocity space $V_{h} \subset\left[H^{1}(\Omega)\right]^{d}$ be the space of continuous piecewise polynomial $\mathbb{R}^{d}$-valued vector fields of order $k$, and let the pressure space $Q_{h} \subset L^{2}(\Omega) \cap C^{0}(\Omega)$ consist of continuous piecewise polynomials of order $l$. To emphasis the order of the underlying polynomials, we occasionally write $V_{h}^{k}$ and $Q_{h}^{l}$.

\subsection{Stabilized Stokes elements}

We recall the definition of consistently stabilized finite element methods for the Stokes problem, following Franca et al. [10, Barth et al. 4]. We first define the bilinear and linear forms

$$
\begin{aligned}
a_{h}\left(\boldsymbol{u}_{h}, \boldsymbol{v}_{h}\right) & =\left(\nabla \boldsymbol{u}_{h}, \nabla \boldsymbol{v}_{h}\right)_{\Omega}, \\
b_{h}\left(\boldsymbol{v}_{h}, p_{h}\right) & =-\left(\nabla \cdot \boldsymbol{v}_{h}, p_{h}\right)_{\Omega}, \\
l_{h}\left(\boldsymbol{v}_{h}\right) & =\left(\boldsymbol{f}, \boldsymbol{v}_{h}\right)_{\Omega} .
\end{aligned}
$$

As categorized by Barth et al. 4], a wide class of consistently stabilized mixed finite element formulation for the Stokes problem can be recast in the following form: find $\left(\boldsymbol{u}_{h}, p_{h}\right) \in V_{h} \times Q_{h}$ such that

$$
A_{h}\left(\boldsymbol{u}_{h}, p_{h} ; \boldsymbol{v}_{h}, q_{h}\right)=L_{h}\left(\boldsymbol{v}_{h}, q_{h}\right) \quad \forall\left(\boldsymbol{v}_{h}, q_{h}\right) \in V_{h} \times Q_{h},
$$

where

$$
\begin{aligned}
A_{h}\left(\boldsymbol{u}_{h}, p_{h} ; \boldsymbol{v}_{h}, q_{h}\right) & =a_{h}\left(\boldsymbol{u}_{h}, \boldsymbol{v}_{h}\right)+b_{h}\left(\boldsymbol{v}_{h}, p_{h}\right)+b_{h}\left(\boldsymbol{u}_{h}, q_{h}\right)-S_{h}\left(\boldsymbol{u}_{h}, p_{h} ; \boldsymbol{v}_{h}, q_{h}\right) \\
L_{h}(\boldsymbol{v}, q) & =l_{h}(\boldsymbol{v})-\delta \sum_{T \in \mathcal{T}} h_{T}^{2}\left(\boldsymbol{f},-\alpha \Delta \boldsymbol{v}_{h}+\beta \nabla q_{h}\right)_{T}
\end{aligned}
$$


and where the stabilization is given by

$$
S_{h}\left(\boldsymbol{u}_{h}, p_{h} ; \boldsymbol{v}_{h}, q_{h}\right)=\delta \sum_{T \in \mathcal{T}} h_{T}^{2}\left(-\Delta \boldsymbol{u}_{h}+\nabla p_{h},-\alpha \Delta \boldsymbol{v}_{h}+\beta \nabla q_{h}\right)_{T}
$$

The parameters $\alpha$ and $\beta$ are chosen from the sets $\{-1,0,1\}$ and $\{-1,1\}$, respectively and $\delta$ denotes some positive constant. Barth et al. 4 point out that the choice $(\alpha, \beta)=(1,1)$ corresponds to the classical scheme introduced by Hughes et al. [16, while on the other hand, the method by Douglas and Wang 8 can be constructed from the parameter choice $(\alpha, \beta)=(-1,1)$. In what follows, we will focus on these two families of stabilized methods for the Stokes problem.

\subsection{A domain decomposition model problem for the Stokes problem}

Let $\Omega=\left(\overline{\Omega_{1} \cup \Omega_{2}}\right)^{\circ}$ be a domain in $\mathbb{R}^{d}$ with Lipschitz boundary $\partial \Omega$, consisting of two (open and bounded) disjoint subdomains $\Omega_{1}$ and $\Omega_{2}$ separated by the interface $\Gamma=\partial \bar{\Omega}_{1} \cap \partial \bar{\Omega}_{2}$. To develop a Nitsche based overlapping mesh method for the Stokes problem, we consider the following domain decomposition model problem for (1.1): find $\boldsymbol{u}: \Omega \rightarrow \mathbb{R}^{3}$ and $p: \Omega \rightarrow \mathbb{R}$ such that

$$
\begin{aligned}
& -\Delta \boldsymbol{u}_{i}+\nabla p_{i}=\boldsymbol{f}_{i} \quad \text { in } \Omega_{i}, \quad i=1,2, \\
& \nabla \cdot \boldsymbol{u}_{i}=0 \quad \text { in } \Omega_{i}, \quad i=1,2, \\
& {[\boldsymbol{u}]=0 \quad \text { on } \Gamma \text {, }} \\
& {\left[\partial_{\boldsymbol{n}} \boldsymbol{u}-p \boldsymbol{n}\right]=0 \quad \text { on } \Gamma \text {, }} \\
& \boldsymbol{u}=0 \quad \text { on } \partial \Omega \text {. }
\end{aligned}
$$

Here and in the following, $v_{i}=\left.v\right|_{\Omega_{i}}$ denotes the restriction of a function or vector field $v$ to a subdomain $\Omega_{i}$. Furthermore, $\boldsymbol{n}$ is the unit normal of $\Gamma$ directed from $\Omega_{2}$ into $\Omega_{1}, \partial_{\boldsymbol{n}} \boldsymbol{u} \equiv \boldsymbol{n} \cdot \nabla \boldsymbol{u}$, and $[v]=v_{2}-v_{1}$ denotes the jump in a function over the interface $\Gamma$.

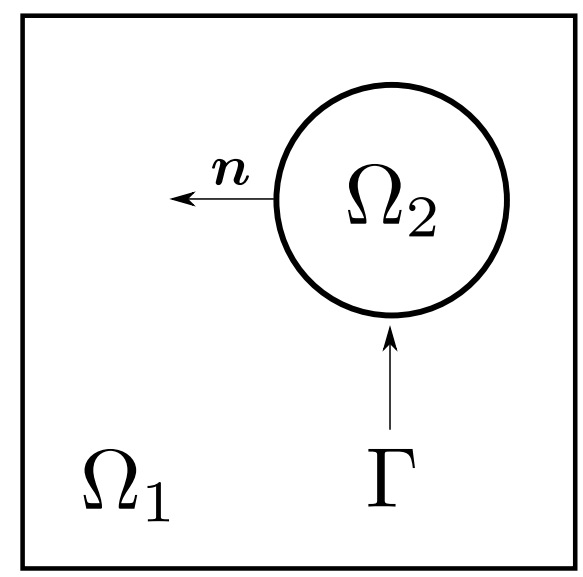

Fig. 2.1 Decomposition of the domain $\Omega$ by introducing the artificial interface $\Gamma$. The weak coupling along the interface $\Gamma$ by the Nitsche method allows independent meshes for the subdomains $\Omega_{1}$ and $\Omega_{2}$, including overlapping meshes.

The decomposition of $\Omega$ into the two subdomains $\Omega_{1}$ and $\Omega_{2}$ motivates the introduction of the broken Sobolev spaces

$$
H^{s}\left(\Omega_{1} \cup \Omega_{2}\right)=H^{s}\left(\Omega_{1}\right) \bigoplus H^{s}\left(\Omega_{2}\right), \quad s \geqslant 0,
$$


endowed with the norm

$$
\|v\|_{s, \Omega_{1} \cup \Omega_{2}}^{2}=\left\|v_{1}\right\|_{s, \Omega_{1}}^{2}+\left\|v_{2}\right\|_{s, \Omega_{2}}^{2} .
$$

The key idea in developing in Nitsche-type methods is to now replace the strong continuity conditions (2.6) and (2.7) by a weak formulation [24, 15, 12, 13, 14. This approach is analogous to that of discontinuous Galerkin methods for elliptic equations [3. Starting with suitable finite element discretizations of $H^{s}\left(\Omega_{1}\right)$ and $H^{s}\left(\Omega_{2}\right)$, a weak formulation can be obtained by multiplying with test functions, integrating by parts and adding certain symmetrization and stabilization terms. A typical example of the resulting interface form will be given as part of the method we present in Section 3 .

We remark that the introduction of the interface $\Gamma$ is purely artificial in our application case and solely serves the purpose of decomposing the domain into suitable subdomains to ease and decouple the subsequent meshing process.

\subsection{Overlapping meshes}

We consider a situation where a background mesh $\mathcal{T}_{0}$ is given for $\Omega=\left(\overline{\Omega_{1} \cup \Omega_{2}}\right)^{\circ}$ and another mesh $\mathcal{T}_{2}$ is given for the overlapping domain $\Omega_{2}$ (see Figure 2.1). Both meshes are assumed to consist of shape-regular simplices $T$. We note that the tessellation $\mathcal{T}_{0}$ of the background domain $\Omega$ may be decomposed into three disjoint subsets:

$$
\mathcal{T}_{0}=\mathcal{T}_{0,1} \cup \mathcal{T}_{0,2} \cup \mathcal{T}_{0, \Gamma},
$$

where $\mathcal{T}_{0,1}=\left\{T \in \mathcal{T}_{0}: T \subset \bar{\Omega}_{1}\right\}, \mathcal{T}_{0,2}=\left\{T \in \mathcal{T}_{0}: T \subset \bar{\Omega}_{2}\right\}$ and $\mathcal{T}_{0, \Gamma}=\left\{T \in \mathcal{T}_{0}:\left|T \cap \Omega_{i}\right|>\right.$ $0, i=1,2\}$ denote the sets of not, completely and partially overlapped elements relative to $\Omega_{2}$, respectively. The meshes $\mathcal{T}_{1}^{*}$ and $\mathcal{T}_{1}$ are then defined by

$$
\begin{aligned}
& \mathcal{T}_{1}^{*}=\mathcal{T}_{0,1} \cup \mathcal{T}_{0, \Gamma}, \\
& \mathcal{T}_{1}=\left\{T \cap \bar{\Omega}_{1}: T \in \mathcal{T}_{1}^{*}\right\} .
\end{aligned}
$$

Moreover, we introduce the tessellated domain $\Omega_{1}^{*}=\bigcup_{T \in \mathcal{T}_{1}^{*}} T$ and the overlap region $\Omega_{\mathrm{O}}=$ $\Omega_{2} \cap \Omega_{1}^{*}$. To ease the notation, we occasionally refer to $\mathcal{T}_{2}$ as $\mathcal{T}_{2}^{*}$ and to $\Omega_{2}$ as $\Omega_{2}^{*}$. See Figure 2.2 for an illustration of the various mesh parts and regions. Furthermore, we introduce the notation $\partial_{e} \mathcal{T}_{j}^{*}$ (the exterior facets) and $\partial_{i} \mathcal{T}_{j}^{*}, j=1,2$ (the interior facets), for the set of facets which belong to either one or two elements, respectively. Here, a facet means an edge in $\mathbb{R}^{2}$ and face in $\mathbb{R}^{3}$. In accordance with $(2.12)$, the sets $\partial_{i} \mathcal{T}_{1}$ and $\partial_{i} \mathcal{T}_{1}$ denote the corresponding set of intersected facets $F \cap \bar{\Omega}_{1}$.

In addition to the shape-regularity, we require that the mesh sizes are compatible over the interface $\Gamma$. More precisely, we assume that there exist a mesh-independent constant $C>0$ such that for all $T \in \mathcal{T}_{0}$ and all $\widetilde{T} \in \mathcal{T}_{2}$ it holds

$$
C^{-1} h_{T} \leqslant h_{\widetilde{T}} \leqslant C h_{T}
$$

whenever $T \cap \widetilde{T} \cap \Gamma \neq \emptyset$. Similar assumptions were made in Hansbo et al. [13.

Finally, we compose suitable finite element spaces for the overlapping meshes. For each mesh $\mathcal{T}_{i}^{*}, i=1,2$, let $\mathcal{V}_{h, i}$ be a finite element space of continuous fixed-order polynomials on $\mathcal{T}_{i}^{*}$. Similar to the broken Sobolev space 2.9 , we introduce the composite finite element space for the whole domain $\Omega$

$$
\mathcal{V}_{h}=\mathcal{V}_{h, 1} \bigoplus \mathcal{V}_{h, 2}
$$

and define the broken Sobolev space norm as before by

$$
\left\|v_{h}\right\|_{s, \mathcal{T}_{1}^{*} \cup \mathcal{T}_{2}}^{2}=\left\|v_{h, 1}\right\|_{s, \mathcal{T}_{1}^{*}}^{2}+\left\|v_{h, 2}\right\|_{s, \mathcal{T}_{2}}^{2} .
$$



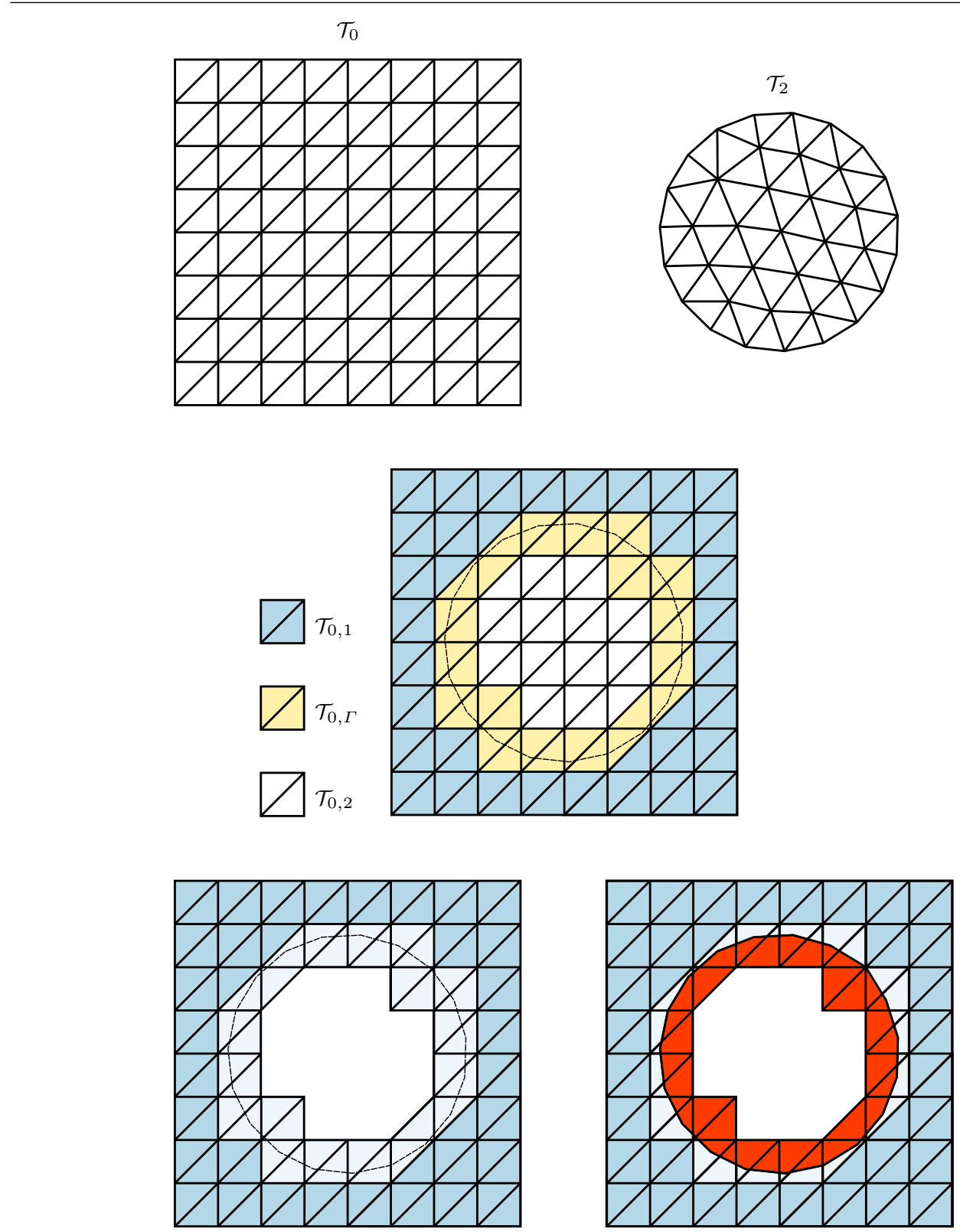

$\ \mathcal{T}_{1}^{*}=\mathcal{T}_{0,1} \cup \mathcal{T}_{0, \Gamma}$

$\mathcal{T}_{1}=\left\{T \cap \Omega_{1}: T \in \mathcal{T}_{1}^{*}\right\}$

$\mathcal{T}_{O}=\left\{T \cap \Omega_{2}: T \in \mathcal{T}_{1}^{*}\right\}$

Fig. 2.2 Summary of notation for overlapping meshes. The starting point is a background mesh $\mathcal{T}_{0}$ and an overlapping mesh $\mathcal{T}_{2}$. The background mesh $\mathcal{T}_{0}$ is then partitioned based on its intersection with the boundary $\Gamma$ of the overlapping domain.

for $v_{h} \in \mathcal{V}_{h}$. Note that both $v_{h, 1}$ and $v_{h, 2}$ contribute to the norm in the overlap region $\Omega_{\mathrm{O}}$. In particular, we define the composite finite element spaces $V_{h}$ and $Q_{h}$ for the velocity and pressure, respectively, by

$$
V_{h}=V_{h, 1}^{k} \bigoplus V_{h, 2}^{k}, \quad Q_{h}=Q_{h, 1}^{l} \bigoplus Q_{h, 2}^{l}
$$




\section{A Nitsche overlapping mesh method for the Stokes problem}

Given a domain $\Omega$, overlapping meshes $\mathcal{T}_{1}^{*}$ and $\mathcal{T}_{2}$ and the composite finite element spaces $V_{h}$ and $Q_{h}$ as introduced in (2.16), we define the bilinear form $A_{h}$ by

$$
\begin{array}{r}
A_{h}\left(\boldsymbol{u}_{h}, p_{h} ; \boldsymbol{v}_{h}, q_{h}\right)=a_{h}\left(\boldsymbol{u}_{h}, \boldsymbol{v}_{h}\right)+b_{h}\left(\boldsymbol{v}_{h}, p_{h}\right)+b_{h}\left(\boldsymbol{u}_{h}, q_{h}\right) \\
+s_{h}\left(\boldsymbol{u}_{h}, \boldsymbol{v}_{h}\right)-S_{h}\left(\boldsymbol{u}_{h}, p_{h} ; \boldsymbol{v}_{h}, q_{h}\right),
\end{array}
$$

where the bilinear forms $a_{h}, b_{h}, s_{h}$ and $S_{h}$ are given by

$$
\begin{aligned}
a_{h}\left(\boldsymbol{u}_{h}, \boldsymbol{v}_{h}\right)= & \left(\nabla \boldsymbol{u}_{h}, \nabla \boldsymbol{v}_{h}\right)_{\Omega_{1} \cup \Omega_{2}}-\left(\left\langle\partial_{\boldsymbol{n}} \boldsymbol{u}_{h}\right\rangle,\left[\boldsymbol{v}_{h}\right]\right)_{\Gamma} \\
& -\left(\left\langle\partial_{\boldsymbol{n}} \boldsymbol{v}_{h}\right\rangle,\left[\boldsymbol{u}_{h}\right]\right)_{\Gamma}+\gamma\left(h^{-1}\left[\boldsymbol{u}_{h}\right],\left[\boldsymbol{v}_{h}\right]\right)_{\Gamma}, \\
b_{h}\left(\boldsymbol{v}_{h}, q_{h}\right)= & -\left(\nabla \cdot \boldsymbol{v}_{h}, q_{h}\right)_{\Omega_{1} \cup \Omega_{2}}+\left(\boldsymbol{n} \cdot\left[\boldsymbol{v}_{h}\right],\left\langle q_{h}\right\rangle\right)_{\Gamma}, \\
s_{h}\left(\boldsymbol{u}_{h}, \boldsymbol{v}_{h}\right)= & \left(\nabla\left(\boldsymbol{u}_{h, 1}-\boldsymbol{u}_{h, 2}\right), \nabla\left(\boldsymbol{v}_{h, 1}-\boldsymbol{v}_{h, 1}\right)\right)_{\Omega_{\mathrm{O}}}, \\
S_{h}\left(\boldsymbol{u}_{h}, p_{h} ; \boldsymbol{v}_{h}, q_{h}\right)= & \delta \sum_{T \in \mathcal{T}_{1}^{*} \cup \mathcal{T}_{2}} h_{T}^{2}\left(-\Delta \boldsymbol{u}_{h}+\nabla p_{h},-\alpha \Delta \boldsymbol{v}_{h}+\beta \nabla q_{h}\right)_{T} .
\end{aligned}
$$

Here, $\langle v\rangle$ denotes a weighted average $\langle v\rangle=\alpha_{1} v_{1}+\alpha v_{2}$ with $\alpha_{1}+\alpha_{2}=1$ for a field $v$ defined on $\Omega$ and discontinuous across the interface $\Gamma$. In accordance with Hansbo et al. [13] and to simplify the presentation, we choose $\langle v\rangle=v_{2}$. Finally, the linear form $L_{h}$ is defined by

$$
L_{h}(\boldsymbol{v}, q)=(\boldsymbol{f}, \boldsymbol{v})-\delta \sum_{T \in \mathcal{T}_{1}^{*} \cup \mathcal{T}_{2}} h_{T}^{2}\left(\boldsymbol{f},-\alpha \Delta \boldsymbol{v}_{h}+\beta \nabla q_{h}\right)_{T} .
$$

With these definitions, the Nitsche based overlapping mesh method for the Stokes problem (1.1) reads: find $\left(\boldsymbol{u}_{h}, p_{h}\right) \in V_{h} \times Q_{h}$ such that

$$
A_{h}\left(\boldsymbol{u}_{h}, p_{h} ; \boldsymbol{v}_{h}, q_{h}\right)=L_{h}\left(\boldsymbol{v}_{h}, q_{h}\right) \quad \forall\left(\boldsymbol{v}_{h}, q_{h}\right) \in V_{h} \times Q_{h} .
$$

Remark 3.1 The forms $s_{h}, S_{h}$ and the mesh $\mathcal{T}_{1}^{*}$ are defined such that contributions from both meshes are taken into account in the overlap region $\Omega_{\mathrm{O}}$. These twice-counted contributions allows us to obtain stability and condition number estimates; this will be discussed further in Section 5 and 7 .

We conclude this section by stating the following Galerkin orthogonality relation.

Proposition 3.1 Let $(\boldsymbol{u}, p) \in\left[H^{2}(\Omega)\right]^{d} \times H^{1}(\Omega)$ be a (weak) solution of 1.1) and $\left(\boldsymbol{u}_{h}, p_{h}\right) \in$ $V_{h} \times Q_{h}$ the solution of the finite element formulation (3.7). Then

$$
A_{h}\left(\boldsymbol{u}-\boldsymbol{u}_{h}, p-p_{h} ; \boldsymbol{v}_{h}, q_{h}\right)=0 \quad \forall\left(\boldsymbol{v}_{h}, q_{h}\right) \in V_{h} \times Q_{h} .
$$

Proof The proof is standard and follows from integration by parts, the continuity conditions 2.6 - 2.7) and observing that $s_{h}\left(\boldsymbol{u}, \boldsymbol{v}_{h}\right) \equiv 0 \equiv S_{h}\left(\boldsymbol{u}, p ; \boldsymbol{v}_{h}, q_{h}\right)$ for all $\left(\boldsymbol{v}_{h}, q_{h}\right) \in V_{h} \times Q_{h}$.

\section{Approximation properties}

In this section, we establish the appropriate interpolation operators and provide interpolation estimates for use in Sections 56 for the a priori error analysis of the method proposed in Section 3 .

With reference to Section 2.4. $\mathcal{V}_{h}$ will here denote some composite finite element space $\mathcal{V}_{h}=$ $\mathcal{V}_{h, 1} \oplus \mathcal{V}_{h, 2}$ comprising piecewise polynomial functions or vector fields defined on $\mathcal{T}_{i}^{*}, i=1,2$. In particular, we shall let $\mathcal{V}_{h}=V_{h}$ or $\mathcal{V}_{h}=Q_{h}$ in the subsequent sections. The constants $C$ involved in the inequalities will depend only on $\Omega_{i}$, the regularity of the function spaces considered, and possibly the shape-regularity of $\mathcal{T}_{i}$ and the polynomial order of $\mathcal{V}_{h, i}, i=1,2$. 


\subsection{Norms}

Given the domain $\Omega=\Omega_{1} \cup \Omega_{2}$ and corresponding overlapping meshes $\mathcal{T}_{1}^{*}$ and $\mathcal{T}_{2}$, we introduce the following pairs of mesh-dependent norms for $(\boldsymbol{v}, q) \in\left[H^{2}(\Omega)\right]^{d} \times H^{1}(\Omega)$ and $\left(\boldsymbol{v}_{h}, q_{h}\right) \in V_{h} \times Q_{h}$ (see 2.16) and 2.15):

$$
\begin{aligned}
\|\boldsymbol{v}\|^{2} & =\|\nabla \boldsymbol{v}\|_{\Omega_{1} \cup \Omega_{2}}^{2}+\left\|\left\langle\partial_{\boldsymbol{n}} \boldsymbol{v}\right\rangle\right\|_{-1 / 2, h, \Gamma}^{2}+\|[\boldsymbol{v}]\|_{1 / 2, h, \Gamma}^{2} \\
\|\boldsymbol{v}\|_{*}^{2} & =\|\nabla \boldsymbol{v}\|_{\mathcal{T}_{1}^{*} \cup \mathcal{T}_{2}}^{2}+\left\|\left\langle\partial_{\boldsymbol{n}} \boldsymbol{v}\right\rangle\right\|_{-1 / 2, h, \Gamma}^{2}+\|[\boldsymbol{v}]\|_{1 / 2, h, \Gamma}^{2} \\
\|q\| \|^{2} & =\|q\|_{\Omega_{1} \cup \Omega_{2}}^{2} \\
\|q\| \|_{*}^{2} & =\|q\|_{\mathcal{T}_{1}^{*} \cup \mathcal{T}_{2}}^{2}
\end{aligned}
$$

where

$$
\|\boldsymbol{v}\|_{\alpha, h, \Gamma}^{2}=\sum_{T \in \mathcal{T}_{2}} h_{T}^{-2 \alpha}\|\boldsymbol{v}\|_{0, \Gamma \cap T}^{2}
$$

and finally

$$
\begin{aligned}
& \left\|\left|(\boldsymbol{v}, q)\left\|\left.\right|^{2}=\right\|\|\boldsymbol{v}\|^{2}+\|||\|^{2},\right.\right. \\
& \|(\boldsymbol{v}, q)\|_{*}^{2}=\|\boldsymbol{v}\|_{*}^{2}+\|q\|_{*}^{2} .
\end{aligned}
$$

Note that $\left.\||\cdot|\|\right|_{*}$-norms are defined on the regular meshes $\mathcal{T}_{1}^{*}$ and $\mathcal{T}_{2}$ and therefore represent proper norms for the discrete finite element functions, while ||$|\cdot|||$ is more suitable for functions defined on $\Omega=\Omega_{1} \cup \Omega_{2}$ only.

\subsection{Trace inequalities and inverse estimates}

In the following, $\mathcal{T}^{*}$ denotes always one of the regular, non-intersected meshes $\mathcal{T}_{1}^{*}$ and $\mathcal{T}_{2}^{*}$. We first recall the following trace inequalities for $v \in H^{1}(\Omega)$ (or $\left.v \in\left[H^{1}(\Omega)\right]^{d}\right)$ [13]:

$$
\begin{aligned}
\|v\|_{\partial T} \leqslant C\left(h_{T}^{-1 / 2}\|v\|_{T}+h_{T}^{1 / 2}\|\nabla v\|_{T}\right) & \forall T \in \mathcal{T}^{*}, \\
\|v\|_{T \cap \Gamma} \leqslant C\left(h_{T}^{-1 / 2}\|v\|_{T}+h_{T}^{1 / 2}\|\nabla v\|_{T}\right) & \forall T \in \mathcal{T}^{*} .
\end{aligned}
$$

The following inverse estimates for $v \in \mathcal{V}_{h}=\mathcal{V}_{h}\left(\mathcal{T}^{*}\right)$ will also be frequently used. See [25, 13, 15] ) for proofs.

$$
\begin{aligned}
h_{T}\left\|\nabla v_{h}\right\|_{T} \leqslant C\left\|v_{h}\right\|_{T} & \forall T \in \mathcal{T}^{*}, \\
h_{F}^{1 / 2}\left\|\partial_{\boldsymbol{n}} v_{h}\right\|_{F} \leqslant C\left\|\nabla v_{h}\right\|_{T} & \forall T \in \mathcal{T}^{*}, \\
h_{F}^{1 / 2}\left\|\partial_{\boldsymbol{n}} v_{h}\right\|_{\Gamma \cap T} \leqslant C\left\|\nabla v_{h}\right\|_{T} & \forall T \in \mathcal{T}^{*} .
\end{aligned}
$$

We emphasize that $h_{F}$ in 4.12 (as in 4.11) denotes the diameter of the entire facet $F$ as part of the mesh $\mathcal{T}^{*}$. Finally, the estimation of the stabilization terms (3.5) will involve the inverse estimates

$$
\begin{array}{ll}
\sum_{T \in \mathcal{T}^{*}} h_{T}^{2}\left\|\nabla v_{h}\right\|^{2} \leqslant C\left\|v_{h}\right\|_{\mathcal{T}^{*}}^{2} \quad & \forall v_{h} \in \mathcal{V}_{h}, \\
\sum_{T \in \mathcal{T}^{*}} h_{T}^{2}\left\|\Delta v_{h}\right\|^{2} \leqslant C\left\|\nabla v_{h}\right\|_{\mathcal{T}^{*}}^{2} & \forall v_{h} \in \mathcal{V}_{h}
\end{array}
$$

To the end of this section, we state a Poincaré type inequality for finite element functions on the overlapped mesh domain $\mathcal{T}_{1}^{*}$. To prove the proposition, we will need the following lemma, which is stated and proved in [23]: 
Lemma 4.1 Let $\mathcal{T}=\{T\}$ be a mesh consisting of shape-regular elements $T$ and take any two elements $T_{1}$ and $T_{2}$ sharing a face $F$. Furthermore, let $u$ be a piecewise polynomial function defined on the macro-element $\mathcal{M}=T_{1} \cup T_{2}$. There exists a constant $C$ depending only on the mesh quality parameters and the maximal polynomial order $p$ of $u$ restricted to each of the elements, such that

$$
\|u\|_{T_{1}}^{2} \leqslant C\left(\|u\|_{T_{2}}^{2}+\sum_{j \leqslant p} h_{F}^{2 j+1}\left(\left[\partial_{\boldsymbol{n}}^{j} u\right],\left[\partial_{\boldsymbol{n}}^{j} u\right]\right)_{F}\right) .
$$

Also note that the inverse inequalities 4.10) and 4.11) can be generalized, by the standard scaling argument, to inverse estimates of the type

$$
\begin{aligned}
h_{T}^{j}\left\|D^{j} u\right\|_{T} & \leqslant C h_{T}^{j-i}\left\|D^{j-i} u\right\|_{T} \quad \text { for } i \leqslant j, \\
h_{F}^{1 / 2}\left\|\partial_{\boldsymbol{n}}^{j} u\right\|_{F} & \leqslant C\left\|D^{j} u\right\|_{T},
\end{aligned}
$$

where $D^{j} u$ denotes the $j$-th total derivative of $u$.

Proposition 4.1 Let $\Omega_{1}$ be the overlapped domain and $\mathcal{T}_{1}^{*}$ be the overlapped mesh, and let $\mathcal{V}_{h, 1}$ be a finite element space on $\mathcal{T}_{1}^{*}$ consisting of continuous piecewise polynomials. Then

$$
\begin{aligned}
& \left\|v_{h}\right\|_{\mathcal{T}_{1}^{*}}^{2} \leqslant C\left(\left\|v_{h}\right\|_{\Omega_{1}}^{2}+\sum_{T \in \mathcal{T}_{1}^{*}} h_{T}^{2}\left\|\nabla v_{h}\right\|_{T}^{2}\right), \\
& \left\|v_{h}\right\|_{\mathcal{T}_{1}^{*}}^{2} \leqslant C\left(\left\|v_{h}\right\|_{\Omega_{1}}^{2}+\left\|\nabla v_{h}\right\|_{\mathcal{T}_{1}^{*}}^{2}\right),
\end{aligned}
$$

for all $v_{h} \in \mathcal{V}_{h, 1}$.

Proof By definition

$$
\left\|v_{h}\right\|_{\mathcal{T}_{1}^{*}}^{2}=\sum_{T \in \mathcal{T}_{0,1}}\left\|v_{h}\right\|_{T}^{2}+\sum_{T_{1} \in \mathcal{T}_{0, \Gamma}}\left\|v_{h}\right\|_{T_{1}}^{2} .
$$

Applying Lemma 4.1 and subsequently invoking 4.17) and 4.16) give

$$
\sum_{T_{1} \in \mathcal{T}_{0, \Gamma}}\left\|v_{h}\right\|_{T_{1}}^{2} \leqslant C \sum_{T \in \mathcal{T}_{1}^{*}}\left(\left\|v_{h}\right\|_{T}^{2}+h_{T}^{2}\left\|\nabla v_{h}\right\|_{T}^{2}\right)
$$

where the shape-regularity of $\mathcal{T}_{1}^{*}$ provides a bound on $h_{F}$ in terms of $h_{T}$. Combining 4.20 and 4.21 yields 4.18).

Since $h_{T} \leqslant\left|\Omega_{1}^{*}\right|$, the second Poincaré type inequality 4.19 is a simple consequence of the first one.

\subsection{Interpolation estimates}

The aim of this section is to construct and analyze an interpolation operator $\pi_{h}: L^{2}(\Omega) \rightarrow \mathcal{V}_{h}$ and a corrected interpolation operator $\pi_{h}^{c}:\left[H^{1}(\Omega)\right]^{d} \rightarrow V_{h}$. The construction is based on extending the standard Scott-Zhang interpolation operator [26].

First, for $s \geqslant 0$, there exists a linear extension operator $\mathcal{E}^{s}: H^{s}\left(\Omega_{1}\right) \rightarrow H^{s}\left(\Omega_{1}^{*}\right)$ and a constant $C>0$ such that $\left.\mathcal{E}^{s} v\right|_{\Omega_{1}}=v$ and

$$
\left\|\mathcal{E}^{s} v\right\|_{s, \Omega_{1}^{*}} \leqslant C\|v\|_{s, \Omega_{1}}
$$

for all $v \in H^{s}\left(\Omega_{1}\right)[27$.

Next, for $i=1,2$, let $\pi_{h, i}: L^{2}\left(\Omega_{i}^{*}\right) \rightarrow \mathcal{V}_{h, i}$ be the Scott-Zhang interpolation operator and recall the standard interpolation error estimates [26]:

$$
\begin{array}{lll}
\left\|v_{i}-\pi_{h, i} v_{i}\right\|_{r, T} \leqslant C h^{s-r}\left|v_{i}\right|_{s, \omega(T)}, & 0 \leqslant r \leqslant s & \forall T \in \mathcal{T}_{i}^{*}, \\
\left\|v_{i}-\pi_{h, i} v_{i}\right\|_{r, F} \leqslant C h^{s-r-1 / 2}\left|v_{i}\right|_{s, \omega(F)}, & 0 \leqslant r \leqslant s & \forall F \in \partial_{i} \mathcal{T}_{i}^{*},
\end{array}
$$

for all $v_{i} \in H^{s}\left(\Omega_{i}^{*}\right)$ for $i=1,2$. Here, $\omega(T)$ is the patch of all elements sharing a vertex with $T$; the patch $\omega(F)$ of a face $F$ is defined analogously. 
Altogether, we define

$$
\pi_{h}: H^{s}\left(\Omega_{1}\right) \bigoplus H^{s}\left(\Omega_{2}\right) \rightarrow \mathcal{V}_{h}, \quad s \geqslant 1,
$$

by

$$
\pi_{h} v=\pi_{h, 1} \mathcal{E}^{s} v_{1} \oplus \pi_{h, 2} v_{2} .
$$

The estimate 4.22 together with the interpolation error estimate 4.23 for the Scott-Zhang interpolation operator imply the following interpolation estimates:

$$
\begin{array}{lll}
\left\|v-\pi_{h} v\right\|_{r, T} \leqslant C h^{s-r}|v|_{s, \omega(T)}, & 0 \leqslant r \leqslant s & \forall T \in \mathcal{T}_{1} \cup \mathcal{T}_{2}, \\
\left\|v-\pi_{h} v\right\|_{r, F} \leqslant C h^{s-r-1 / 2}|v|_{s, \omega(T)}, & 0 \leqslant r \leqslant s & \forall F \in \partial_{i} \mathcal{T}_{1} \cup \partial_{i} \mathcal{T}_{2} .
\end{array}
$$

We now return to our specific finite elements spaces $V_{h}$ and $Q_{h}$. We continue writing $\pi_{h}$ for both the interpolation operator 4.25 defined for $\left[H^{k+1}(\Omega)\right]^{d} \rightarrow V_{h}^{k}$ and $H^{l+1}(\Omega) \rightarrow Q_{h}^{l}$. The following lemma provides an interpolation error estimate in the \|\|$\cdot \| \mid$ norms.

Lemma 4.2 There is a constant $C>0$ such that for all $\boldsymbol{v} \in\left[H^{k+1}(\Omega)\right]^{d}, q \in H^{l+1}(\Omega)$ it holds that

$$
\begin{aligned}
\left\|\boldsymbol{v}-\pi_{h} \boldsymbol{v} \mid\right\| & \leqslant C h^{k}|\boldsymbol{v}|_{k+1, \Omega}, \\
|| q-\pi_{h} q \mid \| & \leqslant C h^{l+1}|q|_{l+1, \Omega}, \\
\left|\left\|\left(\boldsymbol{v}-\pi_{h} \boldsymbol{v}, q-\pi_{h} q\right) \mid\right\|\right. & \leqslant C\left(h^{k}|\boldsymbol{v}|_{k+1, \Omega}+h^{l+1}|q|_{l+1, \Omega}\right) .
\end{aligned}
$$

Proof For the proof of 4.29 we refer to Hansbo et al. [13. The estimate 4.30 follows from the definition of $\pi_{h}$, the interpolation estimate 4.23 and the continuity of the extension operator $\mathcal{E}^{s}$.

We conclude the section by referring to a modified Scott-Zhang interpolation operator introduced by Becker et al. [5] to establish a Nitsche extended finite element method for incompressible elasticity. There, the standard Scott-Zhang interpolant was corrected to gain some additional orthogonality properties on the interface. Although only used for continuous piecewise linear velocity fields in combination with piecewise constant pressures in Becker et al. [5], we will show in the next section that the interpolant can be utilized to prove a sufficient inequality for $b_{h}(\cdot, \cdot)$ adapted to the case of overlapping meshes.

To construct the corrected interpolant, Becker et al. [5] regrouped the elements $T \in \mathcal{T}_{0, \Gamma}$ into a collection $\Gamma_{\omega}$ of patches $\omega_{i}$ in such a way that each patch can be associated with a hat function $\Phi_{i}$ constructed from the finite element functions defined on elements $T \subset \omega_{i}$. Moreover, $\omega_{i}$ and $\Phi_{i}$ have the following three properties: there are constants $c, C>0$ such that

(i) $c h \leqslant \operatorname{diam}\left(\omega_{i}\right) \leqslant C h$

(ii) $c h \leqslant \int_{\Gamma \cap \omega_{i}} \Phi_{i} \leqslant C h$

(iii) $c h^{-1} \leqslant\left|\nabla \Phi_{i}(x)\right| \leqslant C h^{-1}$

Loosely speaking, these properties guarantee that patches and hat functions behave as standard elements and shape functions. Although Becker et al. [5] only elaborate on the construction in two dimension and for a non-fitting interface, a similar construction is possible in any spatial dimension $d$ and for the case where the interface is defined by the boundary of another mesh.

By adjusting the degrees of freedom associated with the hat functions $\left\{\Phi_{i}\right\}$, an interpolation operator

$$
\pi_{h, 1}^{c}:\left[H^{1}\left(\Omega_{1}^{*}\right)\right]^{d} \rightarrow V_{h, 1}
$$

can be defined that satisfies an orthogonality property of the form

$$
\left(\boldsymbol{v}_{1}-\pi_{h, 1}^{c} \boldsymbol{v}_{1}, \boldsymbol{n}\right)_{\Gamma \cap \omega_{i}}=0 \quad \forall \omega_{i},
$$

and an interpolation estimate of the form

$$
\left\|\boldsymbol{v}_{1}-\pi_{h, 1}^{c} \boldsymbol{v}_{1}\right\|_{0, \Omega_{1}^{*}}+h\left\|\nabla\left(\boldsymbol{v}_{1}-\pi_{h, 1}^{c} \boldsymbol{v}_{1}\right)\right\|_{0, \Omega_{1}^{*}} \leqslant C h\|\nabla \boldsymbol{v}\|_{0, \Omega_{1}^{*}} .
$$


Finally, we can construct a corrected Scott-Zhang interpolation operator $\pi_{h}^{c}$ for the composite velocity spaces $V_{h}$ by

$$
\begin{aligned}
\pi_{h}^{c}:\left[H^{1}(\Omega)\right]^{d} & \rightarrow V_{h, 1} \bigoplus V_{h, 2} \\
\pi_{h}^{c}(\boldsymbol{v}) & =\pi_{h, 1}^{c} \mathcal{E}^{1} \boldsymbol{v}_{1} \oplus \pi_{h, 2} \boldsymbol{v}_{2}
\end{aligned}
$$

where $\pi_{h, 2}$ is the standard Scott-Zhang operator on $\Omega_{2}^{*}$.

We will need the following continuity property of the corrected interpolation operator $\pi_{h}^{c}$ with respect to different norms.

Lemma 4.3 Let $\boldsymbol{v} \in\left[H^{1}(\Omega)\right]^{d}$. There exists a constant $C>0$ such that

$$
\left\|\pi_{h}^{c} \boldsymbol{v}\right\|\left\|_{*} \leqslant C\right\| \boldsymbol{v} \|_{1, \Omega_{1} \cup \Omega_{2}} .
$$

Proof By definition,

$$
\left\|\pi_{h}^{c} \boldsymbol{v}_{h}\right\|_{*}^{2}=\left\|\nabla \pi_{h}^{c} \boldsymbol{v}_{h}\right\|_{\mathcal{T}_{1}^{*} \cup \mathcal{T}_{2}}^{2}+\left\|\left\langle\partial_{\boldsymbol{n}} \pi_{h}^{c} \boldsymbol{v}_{h}\right\rangle\right\|_{-1 / 2, h, \Gamma}^{2}+\left\|\left[\pi_{h}^{c} \boldsymbol{v}_{h}\right]\right\|_{1 / 2, h, \Gamma}^{2} .
$$

The estimate for the first term follows directly from the definition (and boundedness) of $\pi_{h}^{c}$ and the continuity of the extension operator $\mathcal{E}$. Using the inverse estimate 4.12 , the bound for the second follows from the bound of the first. To estimate the last term, we first note that $\left\|\left[\pi_{h}^{c} \boldsymbol{v}\right]\right\|_{1 / 2, h, \Gamma}=$ $\left\|\left[\pi_{h}^{c} \boldsymbol{v}-\boldsymbol{v}\right]\right\|_{1 / 2, h, \Gamma}$ since $[\boldsymbol{v}]=0$ for $\boldsymbol{v} \in H^{1}(\Omega)$ and thus

$$
\left\|\left[\pi_{h}^{c} \boldsymbol{v}\right]\right\|_{1 / 2, h, \Gamma}^{2} \leqslant\left\|\pi_{h}^{c} \boldsymbol{v}-\boldsymbol{v}_{1}\right\|_{1 / 2, h, \Gamma}^{2}+\left\|\pi_{h}^{c} \boldsymbol{v}-\boldsymbol{v}_{2}\right\|_{1 / 2, h, \Gamma}^{2} .
$$

Another application of the trace inequality 4.9 combined with interpolation estimates 4.33 yields for $i=1,2$ :

$$
\begin{aligned}
\left\|\pi_{h}^{c} \boldsymbol{v}-\boldsymbol{v}_{i}\right\|_{-1 / 2, h, \Gamma}^{2} & \leqslant \sum_{T \in \mathcal{T}_{i}^{*}} h_{T}^{-1}\left\|\pi_{h}^{c} \boldsymbol{v}-\boldsymbol{v}\right\|_{\Gamma \cap T}^{2} \\
& \leqslant \sum_{T \in \mathcal{T}_{i}^{*}} h^{-1}\left(h^{-1}\left\|\pi_{h}^{c} \boldsymbol{v}-\boldsymbol{v}\right\|_{T}^{2}+h\left\|\nabla\left(\pi_{h}^{c} \boldsymbol{v}-\boldsymbol{v}\right)\right\|_{T}^{2}\right) \\
& \leqslant\|\boldsymbol{v}\|_{1, \mathcal{T}_{i}^{*}}^{2} \leqslant C\|\boldsymbol{v}\|_{1, \Omega_{1} \cup \Omega_{2} .}^{2} .
\end{aligned}
$$

\section{Stability estimates}

In this section, we first prove that the form $a_{h}+s_{h}$ is continuous and coercive with respect to the $\||\cdot|\|_{*}$ norm in Proposition 5.1 that $b_{h}$ is continuous with respect to both the $\||\cdot|\|$ and the $\||\cdot|\|_{*}$ norms in Proposition 5.2 and an additional inequality for $b_{h}$ in Proposition 5.3. Subsequently, these estimates are used to show that the complete stabilized Nitsche form $A_{h}$ satisfies the BabuškaBrezzi inf-sup condition in Theorem 5.1 .

We begin by demonstrating that $a_{h}+s_{h}$ is continuous and stable with respect to the norm ||$\cdot \mid \|_{*}$.

Proposition 5.1 Let $a_{h}$ and $s_{h}$ be defined by (3.2) and 3.4. There are constants $c>0$ and $C>0$ such that

$$
\begin{aligned}
a_{h}\left(\boldsymbol{u}_{h}, \boldsymbol{v}_{h}\right)+s_{h}\left(\boldsymbol{u}_{h}, \boldsymbol{v}_{h}\right) \leqslant C\left\|\boldsymbol{u}_{h}\right\|\left\|_{*}\right\| \boldsymbol{v}_{h} \|_{*} & \forall \boldsymbol{u}_{h}, \boldsymbol{v}_{h} \in V_{h}, \\
c\left\|\boldsymbol{v}_{h}\right\|_{*}^{2} \leqslant a_{h}\left(\boldsymbol{v}_{h}, \boldsymbol{v}_{h}\right)+s_{h}\left(\boldsymbol{v}_{h}, \boldsymbol{v}_{h}\right) & \forall \boldsymbol{v}_{h} \in V_{h} .
\end{aligned}
$$

Proof A proof in the absence of the term $s_{h}$ and with $\mid\|\cdot\|_{*}$ replaced by $\||\cdot|\|$ is given in Hansbo et al. [13. Since by definition $\left\|\boldsymbol{v}_{h}\right\|_{*}^{2}=\|\| \boldsymbol{v}_{h}\|\|^{2}+\left\|\nabla \boldsymbol{v}_{h}\right\|_{\Omega_{\mathrm{O}}}^{2}$ the estimates (5.1) and 5.2) follow directly from the equivalence $\left\|\nabla \boldsymbol{v}_{h}\right\|_{\mathcal{T}_{1}^{*} \cup \mathcal{T}_{2}}^{2} \sim\left\|\nabla \boldsymbol{v}_{h}\right\|_{\Omega_{1} \cup \Omega_{2}}^{2}+\left\|\nabla\left(\boldsymbol{v}_{h, 1}-\boldsymbol{v}_{h, 2}\right)\right\|_{\Omega_{\mathrm{O}}}^{2}$ and the definition of $s_{h}$. 
Next, we state the continuity properties of $b_{h}$ with respect to the various norms.

Proposition 5.2 Let $b_{h}$ be defined by (3.3). There exists a constant $C>0$ such that

$$
\begin{aligned}
b_{h}(\boldsymbol{v}, q) & \leqslant C\|\boldsymbol{v}\|\|\| q \| & & \forall(\boldsymbol{v}, q) \in\left[H^{1}(\Omega)\right]^{3} \times L^{2}(\Omega), \\
b_{h}\left(\boldsymbol{v}_{h}, q_{h}\right) & \leqslant C\left\|\boldsymbol{v}_{h}\right\|_{*}\left\|q_{h}\right\|_{*} & & \forall\left(\boldsymbol{v}_{h}, q_{h}\right) \in V_{h} \times Q_{h} .
\end{aligned}
$$

Proof The first inequality is a trivial consequence of the fact that the interface term $[\boldsymbol{v}]$ vanishes for $\boldsymbol{v} \in\left[H^{1}(\Omega)\right]^{3}$. To prove the second estimate, it is enough to estimate the interface term $\left(\boldsymbol{n} \cdot\left[\boldsymbol{v}_{h}\right],\left\langle q_{h}\right\rangle\right)_{\Gamma}$. Recall that we chose $\left\langle q_{h}\right\rangle=q_{h, 2}$. We combine the Cauchy-Schwarz inequality with 4.9, 4.10) and 4.12 to obtain

$$
\begin{aligned}
\left(\left\langle q_{h}\right\rangle, \boldsymbol{n} \cdot\left[\boldsymbol{v}_{h}\right]\right)_{\Gamma} & \leqslant\left|\left(q_{h, 2}, \boldsymbol{n} \cdot \boldsymbol{v}_{h, 1}\right)_{\Gamma}\right|+\left|\left(q_{h, 2}, \boldsymbol{n} \cdot \boldsymbol{v}_{h, 2}\right)_{\Gamma}\right| \\
& \leqslant\left\|q_{h, 2}\right\|_{-1 / 2, h, \Gamma}\left(\left\|\boldsymbol{v}_{h, 1}\right\|_{1 / 2, h, \Gamma}+\left\|\boldsymbol{v}_{h, 2}\right\|_{1 / 2, h, \Gamma}\right) \\
& \leqslant C\left\|q_{h, 2}\right\|_{\mathcal{T}_{2}}\left(\left\|\nabla \boldsymbol{v}_{h, 1}\right\|_{\mathcal{T}_{1}^{*}}+\left\|\nabla \boldsymbol{v}_{h, 2}\right\|_{\mathcal{T}_{2}}\right) \\
& \leqslant C\left(\left\|q_{h}\right\|_{*}\left\|\boldsymbol{v}_{h}\right\|_{*}\right) .
\end{aligned}
$$

The proof of the inf-sup condition for stabilized Stokes elements often involves an inequality known as Verfürths trick [28. In the next proposition, we present a version adapted to the case of overlapping meshes.

Proposition 5.3 There are constants $C_{1}>0$ and $C_{2}>0$ such that for all $q_{h} \in Q_{h}$ :

$$
\sup _{\boldsymbol{v}_{h} \in V_{h}} \frac{b\left(\boldsymbol{v}_{h}, q_{h}\right)}{\left\|\boldsymbol{v}_{h}\right\|_{*}} \geqslant C_{1}\left\|q_{h}\right\|_{\Omega_{1} \cup \Omega_{2}}-C_{2}\left(\sum_{T \in \mathcal{T}_{1}^{*} \cup \mathcal{T}_{2}} h_{T}^{2}\left\|\nabla q_{h}\right\|_{T}^{2}\right)^{1 / 2} .
$$

Proof Let $q_{h} \in Q_{h}$ be given. The div-operator maps $\left[H^{1}(\Omega)\right]^{d}$ onto $L^{2}(\Omega)$ and there is a constant $C>0$ such that $\|\boldsymbol{v}\|_{1, \Omega} \leqslant C\|\operatorname{div} \boldsymbol{v}\|_{\Omega}$ [11] for all $\boldsymbol{v}$. Choosing $\boldsymbol{v} \in\left[H^{1}(\Omega)\right]^{d}$ such that $\operatorname{div} \boldsymbol{v}=-q_{h}$ thus gives

$$
b_{h}\left(\boldsymbol{v}, q_{h}\right)=\left\|q_{h}\right\|_{\Omega}^{2} \geqslant C^{-1}\left\|q_{h}\right\|_{\Omega}\|\boldsymbol{v}\|_{1, \Omega},
$$

where we have also used the fact that $[\boldsymbol{v}]=0$.

Next, we take $\boldsymbol{v}_{h}=\pi_{h}^{c} \boldsymbol{v}$, where $\pi_{h}^{c}$ is the corrected Scott-Zhang interpolant introduced in Section 4.3, It follows that

$$
\begin{aligned}
b_{h}\left(\boldsymbol{v}_{h}, q_{h}\right) & \equiv b_{h}\left(\pi_{h}^{c} \boldsymbol{v}, q_{h}\right)=b_{h}\left(\pi_{h}^{c} \boldsymbol{v}-\boldsymbol{v}, q_{h}\right)+b\left(\boldsymbol{v}, q_{h}\right) \\
& \geqslant b_{h}\left(\pi_{h}^{c} \boldsymbol{v}-\boldsymbol{v}, q_{h}\right)+C^{-1}\left\|q_{h}\right\|_{\Omega}\|\boldsymbol{v}\|_{1, \Omega} .
\end{aligned}
$$

To proceed further, we recall that $\left\langle q_{h}\right\rangle \equiv q_{h, 2}, \boldsymbol{n} \equiv \boldsymbol{n}_{2}$ and observe that

$$
\sum_{i=1}^{2}\left(\boldsymbol{n}_{i} \cdot\left(\pi_{h, i}^{c} \boldsymbol{v}_{i}-\boldsymbol{v}_{i}\right), q_{h, i}\right)_{\Gamma}=\left(\boldsymbol{n} \cdot\left[\pi_{h}^{c} \boldsymbol{v}-\boldsymbol{v}\right], q_{h, 2}\right)_{\Gamma}+\left(\boldsymbol{n} \cdot\left(\pi_{h, 1}^{c} \boldsymbol{v}_{1}-\boldsymbol{v}_{1}\right),\left[q_{h}\right]\right)_{\Gamma} .
$$

Using this identity and integrating the first term in (5.8) by parts, we derive that

$$
\begin{aligned}
b_{h}\left(\pi_{h}^{c} \boldsymbol{v}-\boldsymbol{v}, q_{h}\right) & \equiv-\left(\nabla \cdot\left(\pi_{h}^{c} \boldsymbol{v}-\boldsymbol{v}\right), q_{h}\right)_{\Omega_{1} \cup \Omega_{2}}+\left(\boldsymbol{n} \cdot\left[\pi_{h}^{c} \boldsymbol{v}-\boldsymbol{v}\right], q_{h, 2}\right)_{\Gamma} \\
& =\underbrace{\sum_{i=1}^{2} \sum_{T \in \mathcal{T}_{i}}\left(\pi_{h}^{c} \boldsymbol{v}-\boldsymbol{v}, \nabla q_{h}\right)_{T \cap \Omega_{i}}}_{I}-\underbrace{\left(\boldsymbol{n} \cdot\left(\pi_{h, 1}^{c} \boldsymbol{v}_{1}-\boldsymbol{v}_{1}\right),\left[q_{h}\right]\right)_{\Gamma}}_{I I} .
\end{aligned}
$$

Rewriting $\left(\pi_{h}^{c} \boldsymbol{v}-\boldsymbol{v}, \nabla q_{h}\right)_{T \cap \Omega}=\left(h_{T}^{-1}\left(\pi_{h}^{c} \boldsymbol{v}-\boldsymbol{v}\right), h_{T} \nabla q_{h}\right)_{T \cap \Omega}$ and applying the interpolation estimates 4.27) and 4.33), the first term $I$ can be bounded from below by

$$
|I| \geqslant-\|\nabla \boldsymbol{v}\|_{\mathcal{T}_{1}^{*} \cup \mathcal{T}_{2}}\left(\sum_{T \in \mathcal{T}_{1}^{*} \cup \mathcal{T}_{2}} h_{T}^{2}\left\|\nabla q_{h}\right\|^{2}\right)^{1 / 2}
$$


To estimate the second term $I I$, we exploit the orthogonality property 4.32 and write

$$
\begin{aligned}
\left(\boldsymbol{n} \cdot\left(\pi_{h, 1}^{c} \boldsymbol{v}_{1}-\boldsymbol{v}_{1}\right),\left[q_{h}\right]\right)_{\Gamma}= & \sum_{\omega \in \Gamma_{\omega}}\left(\boldsymbol{n} \cdot\left(\pi_{h, 1}^{c} \boldsymbol{v}_{1}-\boldsymbol{v}_{1}\right), q_{h, 2}-\bar{q}_{h, 2}\right)_{\Gamma \cap \omega} \\
& -\sum_{\omega \in \Gamma_{\omega}}\left(\boldsymbol{n} \cdot\left(\pi_{h, 1}^{c} \boldsymbol{v}_{1}-\boldsymbol{v}_{1}\right), q_{h, 1}-\bar{q}_{h, 1}\right)_{\Gamma \cap \omega}
\end{aligned}
$$

where $\left.\bar{q}_{h, i}\right|_{\omega}=\frac{1}{|\omega|} \int_{\omega} q_{h, i} \mathrm{~d} x, i=1,2$. Now each contribution in the sum 5.9 can bounded by

$$
\begin{aligned}
\left(\boldsymbol{n} \cdot\left(\pi_{h, 1}^{c} \boldsymbol{v}_{1}-\boldsymbol{v}_{1}\right)\right. & \left., q_{h, i}-\bar{q}_{h, i}\right)_{\Gamma \cap \omega} \\
& \leqslant\left\|\boldsymbol{n} \cdot\left(\pi_{h, 1}^{c} \boldsymbol{v}_{1}-\boldsymbol{v}_{1}\right)\right\|_{1 / 2, h, \Gamma \cap \omega}\left\|q_{h, i}-\bar{q}_{h, i}\right\|_{-1 / 2, h, \Gamma \cap \omega}
\end{aligned}
$$

Using again the trace inequality 4.9 , the last factor can estimated by

$$
\left\|q_{h, i}-\bar{q}_{h, i}\right\|_{-1 / 2, h, \Gamma \cap \omega} \leqslant C|\omega|\left\|\nabla q_{h, i}\right\|_{\omega} \leqslant C h_{T}\left\|\nabla q_{h, i}\right\|_{\omega}
$$

since $q_{h, i}-\bar{q}_{h, i}$ has mean value zero on $\omega$ and so $\left\|q_{h, i}-\bar{q}_{h, i}\right\|_{\omega} \leqslant|\omega|\left\|\nabla q_{h, i}\right\|_{\omega}$. To arrive at an estimate for first factor in (5.10), we use the trace inequality $\sqrt{4.9}$ in combination with the interpolation estimate 4.33 and the continuity of the extension operator:

$$
\left\|\boldsymbol{n} \cdot\left(\pi_{h, 1}^{c} \boldsymbol{v}_{1}-\boldsymbol{v}_{1}\right)\right\|_{1 / 2, h, \Gamma \cap \omega} \leqslant\left\|\nabla \boldsymbol{v}_{1}\right\|_{\omega \cap \Omega_{1}} .
$$

Thus

$$
\begin{aligned}
\left|\left(\boldsymbol{n} \cdot\left(\pi_{h, 1}^{c} \boldsymbol{v}_{1}-\boldsymbol{v}_{1}\right),\left[q_{h}\right]\right)_{\Gamma}\right| & \leqslant \sum_{\omega \in \Gamma_{\omega}} h_{T}\left(\left\|\nabla q_{h, 1}\right\|_{\omega}+\left\|\nabla q_{h, 2}\right\|_{\omega}\right)\left\|\nabla \boldsymbol{v}_{1}\right\|_{\omega \cap \Omega_{1}} \\
& \leqslant C\left(\sum_{T \in \mathcal{T}_{1}^{*} \cup \mathcal{T}_{2}} h_{T}^{2}\left\|\nabla q_{h}\right\|_{T}^{2}\right)^{1 / 2}\|\nabla \boldsymbol{v}\|_{\Omega_{1}} .
\end{aligned}
$$

Putting $I$ and $I I$ together, we see that

$$
\left|b_{h}\left(\pi_{h}^{c} \boldsymbol{v}-\boldsymbol{v}, q_{h}\right)\right| \leqslant C\left(\sum_{T \in \mathcal{T}_{1}^{*} \cup \mathcal{T}_{2}} h_{T}^{2}\left\|\nabla q_{h}\right\|_{T}^{2}\right)^{1 / 2}\|\nabla \boldsymbol{v}\|_{\Omega},
$$

which together with 5.8 gives the estimate

$$
\frac{b\left(\pi_{h}^{c} \boldsymbol{v}, q_{h}\right)}{\|\boldsymbol{v}\|_{\Omega}} \geqslant \widetilde{C}_{1}\left\|q_{h}\right\|_{\Omega_{1} \cup \Omega_{2}}-\widetilde{C}_{2}\left(\sum_{T \in \mathcal{T}_{1}^{*} \cup \mathcal{T}_{2}} h_{T}^{2}\left\|\nabla q_{h}\right\|_{T}^{2}\right)^{1 / 2}
$$

Now the estimate follows from the continuity 4.35 of the operator $\pi_{h}^{c}$.

Remark 5.1 Although looking very similar to the standard "bad-inequality", the inequality (5.5) bears some subtle differences. Note that we count contributions $\left\|\nabla q_{h}\right\|_{T}$ twice in the overlap domain $\Omega_{O}$. This is mainly a consequence of the trace inequality 4.9 . The $L^{2}$-norm of $q_{h}$ on the other hand is only taken on $\Omega_{1} \cup \Omega_{2}$; thus requiring special consideration in the subsequent proof of the inf-sup estimate for $A_{h}$ using the norm $\left\|\left|q_{h}\right|\right\|_{*}=\left\|q_{h}\right\|_{\mathcal{T}_{1}^{*} \cup \mathcal{T}_{2}}$.

We conclude the section by proving the inf-sup condition for the bilinear form $A_{h}$ with respect to the norm $\|||(\cdot, \cdot) \mid\|_{*}$.

Theorem 5.1 Let $A_{h}$ be defined by 3.1 -3.5 and assume that either $\{\alpha, \beta\}=\{1,1\}$ or $\{\alpha, \beta\}=$ $\{-1,1\}$. Then there is a constant $c$ such that

$$
\sup _{\left(\boldsymbol{v}_{h}, q_{h}\right) \in V_{h} \times Q_{h}} \frac{A_{h}\left(\boldsymbol{u}_{h}, p_{h} ; \boldsymbol{v}_{h}, q_{h}\right)}{\left\|\left(\boldsymbol{v}_{h}, q_{h}\right)\right\| \|_{*}} \geqslant c\left\|\mid\left(\boldsymbol{u}_{h}, p_{h}\right)\right\|_{*} \quad \forall\left(\boldsymbol{u}_{h}, p_{h}\right) \in V_{h} \times Q_{h} .
$$


Proof The proof follows the presentation given in Franca et al. [10. Let $\left(\boldsymbol{u}_{h}, p_{h}\right)$ be given and consider the case $\{\alpha, \beta\}=\{1,1\}$.

Step 1: Choose $\boldsymbol{v}_{h}=-\boldsymbol{w}$ such that the supremum in 5.5 is attained. By scaling $\boldsymbol{w}$, we can assume that $\left|\|\boldsymbol{w} \mid\|_{*}=\left\|p_{h}\right\|_{\Omega_{1} \cup \Omega_{2}}\right.$. Inserting $\left(\boldsymbol{v}_{h}, q_{h}\right)=(-\boldsymbol{w}, 0)$ into $A_{h}$, we obtain via (5.1) and 5.5):

$$
\begin{aligned}
A_{h}\left(\boldsymbol{u}_{h}, p_{h} ; \boldsymbol{v}_{h}, q_{h}\right)= & -a_{h}\left(\boldsymbol{u}_{h}, \boldsymbol{w}\right)-s_{h}\left(\boldsymbol{u}_{h}, \boldsymbol{w}\right)-b_{h}\left(\boldsymbol{w}, p_{h}\right)+S_{h}\left(\boldsymbol{u}_{h}, p_{h} ; \boldsymbol{w}, 0\right) \\
\geqslant & -C_{a}\|\| \boldsymbol{u}_{h}\|\|_{*}\left\|p_{h}\right\|_{\Omega_{1} \cup \Omega_{2}} \\
& +\left\{C_{1}\left\|p_{h}\right\|_{\Omega_{1} \cup \Omega_{2}}-C_{2}\left(\sum_{T \in \mathcal{T}_{1}^{*} \cup \mathcal{T}_{2}} h_{T}^{2}\left\|\nabla q_{h}\right\|_{T}^{2}\right)^{1 / 2}\right\}\left\|p_{h}\right\|_{\Omega_{1} \cup \Omega_{2}} \\
& +\delta \underbrace{\delta \sum_{T \in \mathcal{T}_{1}^{*} \cup \mathcal{T}_{2}} h_{T}^{2}\left(-\Delta \boldsymbol{u}_{h}+\nabla p_{h},-\Delta \boldsymbol{w}\right)_{T}}_{I} .
\end{aligned}
$$

We estimate $I$ using Cauchy-Schwarz and the inverse inequality (4.14):

$$
\begin{aligned}
I & \geqslant-C_{I} \delta\|\nabla \boldsymbol{w}\|_{\mathcal{T}_{1}^{*} \cup \mathcal{T}_{2}}\left(\left\|\nabla \boldsymbol{u}_{h}\right\|_{\mathcal{T}_{1}^{*} \cup \mathcal{T}_{2}}+\left(\sum_{T \in \mathcal{T}_{1}^{*} \cup \mathcal{T}_{2}} h_{T}^{2}\left\|\nabla p_{h}\right\|_{T}\right)^{1 / 2}\right) \\
& \geqslant-C_{I} \delta\left\|p_{h}\right\|_{\Omega_{1} \cup \Omega_{2}}\left(\left\|\boldsymbol{u}_{h}\right\|_{*}+\left(\sum_{T \in \mathcal{T}_{1}^{*} \cup \mathcal{T}_{2}} h_{T}^{2}\left\|\nabla p_{h}\right\|_{T}\right)^{1 / 2}\right)
\end{aligned}
$$

where the last inequality above follows as $\|\nabla \boldsymbol{v}\|_{\mathcal{T}_{1}^{*} \cup \mathcal{T}_{2}} \leqslant\|\boldsymbol{v}\|_{*}$ for all $\boldsymbol{v}$ and $\|\boldsymbol{w}\|\left\|_{*}=\right\| p_{h} \|_{\Omega_{1} \cup \Omega_{2}}$. Inserting this final estimate for $I$ into (5.12) and using the inequality $a b \leqslant \epsilon a^{2}+(4 \epsilon)^{-1} b^{2}$ for any $\epsilon>0$, we arrive at

$$
\begin{aligned}
A_{h}\left(\boldsymbol{u}_{h}, p_{h} ;-\boldsymbol{w}, 0\right) \geqslant & \left(C_{1}-\epsilon\left(C_{a}+C_{2}+\delta C_{I}\right)\right)\left\|p_{h}\right\|_{\Omega_{1} \cup \Omega_{2}}^{2} \\
& -\left(C_{a}+\delta C_{I}\right)(4 \epsilon)^{-1}\left\|\boldsymbol{u}_{h}\right\|_{*}^{2} \\
& -\left(C_{2}+\delta C_{I}\right)(4 \epsilon)^{-1} \sum_{T \in \mathcal{T}_{1}^{*} \cup \mathcal{T}_{2}} h_{T}^{2}\left\|\nabla p_{h}\right\|_{T}^{2} \\
\geqslant & \widetilde{C}_{1}\left\|p_{h}\right\|_{\Omega_{1} \cup \Omega_{2}}^{2}-\widetilde{C}_{2}\|\| \boldsymbol{u}_{h}\left\|_{*}^{2}-\widetilde{C}_{3} \sum_{T \in \mathcal{T}_{1}^{*} \cup \mathcal{T}_{2}} h_{T}^{2}\right\| \nabla p_{h} \|_{T}^{2}
\end{aligned}
$$

with positive constants $\widetilde{C}_{1}, \widetilde{C}_{2}, \widetilde{C}_{3}$ for an appropriate choice of $\epsilon$.

Step 2: Now choose $\left(\boldsymbol{v}_{h}, q_{h}\right)=\left(\boldsymbol{u}_{h},-p_{h}\right)$. Proposition 5.1 and subsequently 4.14 give that

$$
\begin{aligned}
A_{h}\left(\boldsymbol{u}_{h}, p_{h} ; \boldsymbol{u}_{h},-p_{h}\right) & =a_{h}\left(\boldsymbol{u}_{h}, \boldsymbol{u}_{h}\right)+s_{h}\left(\boldsymbol{u}_{h}, \boldsymbol{u}_{h}\right)-S_{h}\left(\boldsymbol{u}_{h}, p_{h} ; \boldsymbol{u}_{h},-p_{h}\right) \\
& \geqslant c_{a}\|\| \boldsymbol{u}_{h} \|_{*}^{2}-\delta \sum_{T \in \mathcal{T}_{1}^{*} \cup \mathcal{T}_{2}} h_{T}^{2}\left(-\Delta \boldsymbol{u}_{h}+\nabla p_{h},-\Delta \boldsymbol{u}_{h}-\nabla p_{h}\right)_{T} \\
& \geqslant\left(c_{a}-\delta C_{I}\right)\left\|\boldsymbol{u}_{h}\right\|_{*}^{2}+\delta \sum_{T \in \mathcal{T}_{1}^{*} \cup \mathcal{T}_{2}} h_{T}^{2}\left\|\nabla p_{h}\right\|_{T}^{2} \\
& \geqslant \widetilde{C}\left\|\boldsymbol{u}_{h}\right\|_{*}^{2}+\delta \sum_{T \in \mathcal{T}_{1}^{*} \cup \mathcal{T}_{2}} h_{T}^{2}\left\|\nabla p_{h}\right\|_{T}^{2}
\end{aligned}
$$

with positive $\widetilde{C}$ as long as $0<\delta<c_{a} C_{I}^{-1}$.

Step 3: To complete the proof, we combine step 1 and step 2 by taking $\left(\boldsymbol{v}_{h}, q_{h}\right)=\left(\boldsymbol{u}_{h}-\eta \boldsymbol{w},-p_{h}\right)$ for some $\eta>0$. By choosing $\eta$ sufficiently small,

$$
\begin{aligned}
A\left(\boldsymbol{u}_{h}, p_{h} ; \boldsymbol{v}_{h}, q_{h}\right) & \geqslant C_{1}\left\|\boldsymbol{u}_{h}\right\|_{*}^{2}+C_{2}\left\|p_{h}\right\|_{\Omega_{1} \cup \Omega_{2}}^{2}+C_{3} \sum_{T \in \mathcal{T}_{1}^{*} \cup \mathcal{T}_{2}} h_{T}^{2}\left\|\nabla p_{h}\right\|_{T}^{2} \\
& \geqslant C_{1}\left\|\boldsymbol{u}_{h}\right\|_{*}^{2}+\widetilde{C}_{2}\left\|p_{h}\right\|_{*}^{2} \geqslant \min \left(C_{1}, \widetilde{C}_{2}\right)\left\|\left(\boldsymbol{u}_{h}, p_{h}\right)\right\|_{*}^{2},
\end{aligned}
$$


for some constants $C_{1}, C_{2}, C_{3}>0$ where we used Proposition 4.1 to find that for some constant $\widetilde{C}_{2}>0$

$$
C_{2}\left\|p_{h}\right\|_{\Omega_{1} \cup \Omega_{2}}^{2}+C_{3} \sum_{T \in \mathcal{T}_{1}^{*} \cup \mathcal{T}_{2}} h_{T}^{2}\left\|\nabla p_{h}\right\|_{T}^{2} \geqslant \widetilde{C}_{2}\left\|p_{h}\right\| \|_{*}^{2} .
$$

Since $\left\|\mid\left(\boldsymbol{v}_{h}, q_{h}\right)\right\|_{*} \leqslant\left\|\left(\boldsymbol{u}_{h}, p_{h}\right)\right\|\left\|_{*}+\eta\right\|\|\boldsymbol{w}\|_{*} \leqslant(1+\eta)\left\|\left(\boldsymbol{u}_{h}, p_{h}\right)\right\| \|_{*}$, the estimate (5.11) follows.

The proof for $\{\alpha, \beta\}=\{-1,1\}$ differs only in the derivation of the final estimate in step 2 and can be taken from Franca et al. [10] with the presented adaption to the overlapping meshes formulation.

Remark 5.2 We would like to comment on the role of the different ghost-penalties. As remarked earlier, the bad-inequality 5.5 "mixes domains" in the sense that it the contributions $\left\|\nabla q_{h}\right\|_{T}$ are taken from the elements in $\mathcal{T}_{1}^{*} \cup \mathcal{T}_{2}$, so that the contributions from the overlap region $\Omega_{\mathrm{O}}$ are counted twice, while $\left\|q_{h}\right\|$ is evaluated only once on $\Omega_{1} \cup \Omega_{2}$. The role of the pressure terms in the least-squares ghost-penalty $\sum_{T \in \mathcal{T}_{1}^{*} \cup \mathcal{T}_{2}} h_{T}^{2}\left(-\Delta \boldsymbol{u}_{h}+\nabla p_{h},-\Delta \boldsymbol{v}_{h}+\nabla q_{h}\right)_{T}$ is two-fold. First, they compensate the negative contributions $-\sum_{T \in \mathcal{T}_{1}^{*}} h_{T}^{2}\left\|\nabla q_{h}\right\|_{T}^{2}$ in the bad-inequality. Secondly, they allow in combination with Lemma 4.1 to pass from $\left\|q_{h}\right\|_{\Omega_{1} \cup \Omega_{2}}$ to $\left\|q_{h}\right\|_{\mathcal{T}_{1}^{*} \cup \mathcal{T}_{2}}$. We further note that the velocity terms in the least-squares ghost-penalty make it necessary (via the inverse inequality 4.14) to control $\left\|\nabla \boldsymbol{u}_{h, 1}\right\|_{\Omega_{\mathrm{O}}}$. This is precisely the role of $s_{h}\left(\boldsymbol{u}_{h}, \boldsymbol{v}_{h}\right)=\left(\nabla\left(\boldsymbol{u}_{h, 1}-\right.\right.$ $\left.\left.\boldsymbol{u}_{h, 2}\right), \nabla\left(\boldsymbol{v}_{h, 1}-\boldsymbol{v}_{h, 2}\right)\right)_{\Omega_{\mathrm{O}}}$.

\section{A priori error estimate}

The previous results on the interpolation errors, the Galerkin orthogonality of the discretization and its stability enable us to state the following a priori estimate for the error in the discrete solution.

Theorem 6.1 Let $k, l \geqslant 1$ and $(\alpha, \beta)=( \pm 1,1)$. Assume that $(\boldsymbol{u}, p) \in\left[H^{k+1}(\Omega)\right]^{d} \times H^{l+1}(\Omega)$ is a (weak) solution of the Stokes problem 1.1. Then the finite element solution $\left(\boldsymbol{u}_{h}, p_{h}\right) \in V_{h}^{k} \times Q_{h}^{l}$ defined in (3.7) satisfies the following error estimate:

$$
\|\left|\left(\boldsymbol{u}-\boldsymbol{u}_{h}, p-p_{h}\right)\right| \mid \leqslant C\left(h^{k}|\boldsymbol{u}|_{k+1, \Omega}+h^{l+1}|p|_{l+1, \Omega}\right) .
$$

Proof By applying the triangle inequality, we have

$$
\left\|\left(\boldsymbol{u}-\boldsymbol{u}_{h}, p-p_{h}\right)\right\||\leqslant|\left\|\left(\boldsymbol{u}-\pi_{h} \boldsymbol{u}, p-\pi_{h} p\right)\right\||+|\left\|\left(\pi_{h} \boldsymbol{u}-\boldsymbol{u}_{h}, \pi_{h} p-p_{h}\right)\right\|_{*} .
$$

Given the interpolation estimate 4.31), it is suffices to bound the discretization error $\left(\pi_{h} \boldsymbol{u}-\right.$ $\left.\boldsymbol{u}_{h}, \pi_{h} p-p\right)$. By Theorem 5.1 there exists a $\left(\boldsymbol{v}_{h}, p_{h}\right)$ such that $\left\|\mid\left(\boldsymbol{v}_{h}, p_{h}\right)\right\| \|_{*} \leqslant 1$ and

$$
c\left\|\mid\left(\pi_{h} \boldsymbol{u}-\boldsymbol{u}_{h}, \pi_{h} p-p_{h}\right)\right\|_{*} \leqslant A_{h}\left(\boldsymbol{u}_{h}-\pi_{h} \boldsymbol{u}, p-\pi_{h} p ; \boldsymbol{v}_{h}, q_{h}\right) .
$$

Thus, the Galerkin orthogonality 3.8 and the definition of $A_{h}$ give

$$
\begin{aligned}
c\left\|\left(\pi_{h} \boldsymbol{u}-\boldsymbol{u}_{h}, \pi_{h} p-p_{h}\right)\right\|_{*} \leqslant & A_{h}\left(\boldsymbol{u}-\pi_{h} \boldsymbol{u}, p-\pi_{h} p ; \boldsymbol{v}_{h}, q_{h}\right) \\
= & \underbrace{a_{h}\left(\boldsymbol{u}-\pi_{h} \boldsymbol{u}, \boldsymbol{v}_{h}\right)+s_{h}\left(\boldsymbol{u}-\pi_{h} \boldsymbol{u}, \boldsymbol{v}_{h}\right)}_{I} \\
& +\underbrace{b_{h}\left(\boldsymbol{v}_{h}, p-\pi_{h} p\right)}_{I I}+\underbrace{b_{h}\left(\boldsymbol{u}-\pi_{h} \boldsymbol{u}, q_{h}\right)}_{I I I} \\
& \underbrace{-S_{h}\left(\boldsymbol{u}-\pi_{h} \boldsymbol{u} ; p-\pi_{h} p, \boldsymbol{v}_{h}, q_{h}\right)}_{I V} .
\end{aligned}
$$

To estimate the first term, we use (5.1) and the interpolation estimate 4.29 to obtain

$$
|I| \leqslant C\left|\left\|\boldsymbol{u}-\left.\pi_{h} \boldsymbol{u}\left|\|\|_{*}\left\|\boldsymbol{v}_{h}\right\|_{*} \leqslant C h^{k}\right| \boldsymbol{u}\right|_{k+1, \Omega}\left|\left\|\left.\boldsymbol{v}_{h}\left|\|_{*} \leqslant C h^{k}\right| \boldsymbol{u}\right|_{k+1, \Omega} .\right.\right.\right.\right.
$$


Recalling that $\langle p\rangle=p_{2}$, the second term $I I$ can be estimated by

$$
\begin{aligned}
|I I| & =\left|\left(\nabla \cdot \boldsymbol{v}, p-\pi_{h} p\right)_{\Omega_{1} \cup \Omega_{2}}+\left(\boldsymbol{n} \cdot[\boldsymbol{v}],\left\langle p-\pi_{h} p\right\rangle\right)_{\Gamma}\right| \\
& \leqslant\|\boldsymbol{v}\|\left|\left\|p-\pi_{h} p\right\|\right|+\|[\boldsymbol{v}]\|_{1 / 2, h, \Gamma}\left\|p_{2}-\pi_{h, 2} p_{2}\right\|_{-1 / 2, h, \Gamma} \\
& \leqslant\|\boldsymbol{v}\|\left|\left\|\left|\left\|p-\pi_{h} p \mid\right\|+C\|[\boldsymbol{v}]\|_{1 / 2, h, \Gamma}\left(\left\|p_{2}-\pi_{h, 2} p_{2}\right\|_{\Omega_{2}}+h\left\|\nabla\left(p_{2}-\pi_{h, 2} p_{2}\right)\right\|_{\Omega_{2}}\right)\right.\right.\right. \\
& \leqslant\left. C\|\boldsymbol{v}\|\left|h^{l+1}\right| p\right|_{l+1, \Omega} \leqslant C h^{l+1}|p|_{l+1, \Omega},
\end{aligned}
$$

where we used the trace inequality 4.8 in the penultimate step and finally 4.10 in combination with the interpolation estimate 4.30). The third term can be treated similarly to obtain

$$
|I I I| \leqslant C h^{k+1}|\boldsymbol{u}|_{k+1, \Omega}||\left|q_{h}\right| \|_{*} \leqslant C h^{k+1}|\boldsymbol{u}|_{k+1, \Omega} .
$$

For the last term $I V$, several applications of Cauchy-Schwarz yield

$$
\begin{aligned}
|I V|= & \delta\left|\sum_{T \in \mathcal{T}_{1}^{*} \cup \mathcal{T}_{2}} h_{T}^{2}\left(-\Delta\left(\boldsymbol{u}-\pi_{h} \boldsymbol{u}\right),+\nabla\left(p-\pi_{h} p\right), \pm \Delta \boldsymbol{v}_{h}+\nabla q_{h}\right)_{T}\right| \\
\leqslant & C\left(\sum_{T \in \mathcal{T}_{1}^{*} \cup \mathcal{T}_{2}} h_{T}^{2}\left\|\Delta\left(\boldsymbol{u}-\pi_{h} \boldsymbol{u}\right)\right\|_{\mathcal{T}_{1}^{*} \cup \mathcal{T}_{2}}^{2}+h_{T}^{2}\left\|\nabla\left(p-\pi_{h} p\right)\right\|_{\mathcal{T}_{1}^{*} \cup \mathcal{T}_{2}}^{2}\right)^{1 / 2} \\
& \times\left(\sum_{T \in \mathcal{T}_{1}^{*} \cup \mathcal{T}_{2}} h_{T}^{2}\left\|\Delta \boldsymbol{v}_{h}\right\|_{\mathcal{T}_{1}^{*} \cup \mathcal{T}_{2}}^{2}+h_{T}^{2}\left\|\nabla q_{h}\right\|_{\mathcal{T}_{1}^{*} \cup \mathcal{T}_{2}}^{2}\right)^{1 / 2}
\end{aligned}
$$

After applying the inverse inequalities 4.14 and 4.13 to the second term, the interpolation estimate 4.27 implies

$$
|I V| \leqslant C\left(h^{k}|\boldsymbol{u}|_{k+1, \Omega}+h^{l+1}|p|_{l+1, \Omega}\right)||\left|\left(\boldsymbol{v}_{h}, q_{h}\right)\right||| \leqslant C\left(h^{k}|\boldsymbol{u}|_{k+1, \Omega}+h^{l+1}|p|_{l+1, \Omega}\right) .
$$

Summing up, we obtain that

$$
\begin{aligned}
\|\left|\left(\boldsymbol{u}-\boldsymbol{u}_{h}, p-p_{h}\right)\right||| & \leqslant||\left|\left(\boldsymbol{u}-\pi_{h} \boldsymbol{u}, p-\pi_{h} p\right)\right||+| I|+| I I|+| I I I|+| I V \mid \\
& \leqslant C\left(h^{k}|\boldsymbol{u}|_{k+1, \Omega}+h^{l+1}|p|_{l, \Omega}\right),
\end{aligned}
$$

which concludes the proof.

\section{Condition number estimate}

To conclude the analysis of the discretization presented in Section 3 , we here show that the condition number of the associated stiffness matrix is uniformly bounded by $\mathrm{Ch}^{-2}$ independently of the location of the overlapping mesh $\mathcal{T}_{2}$. The proof of the condition number estimate follows the approach of Ern and Guermond [9].

Let $\left(\boldsymbol{v}_{h}, q_{h}\right)=\sum_{i=1}^{N} V_{i} \varphi_{i}$ where $\left\{\varphi_{i}\right\}_{i=1}^{N}$ is a basis for the element space $V_{h} \times Q_{h} ; V=\left\{V_{i}\right\}_{i}$ thus denotes the expansion coefficients of $\left(\boldsymbol{v}_{h}, q_{h}\right)$ in the given basis. Similarly, let $W$ label the expansion coefficients of fields denoted $\left(\boldsymbol{w}_{h}, r_{h}\right)$. Denote the inner product in $\mathbb{R}^{N}$ by $(V, W)_{N}=\sum_{i=1}^{N} V_{i} W_{i}$ and the corresponding norm by $|V|_{N}^{2}=(V, V)_{N}$. The stiffness matrix $\mathcal{A}$ associated with the form 3.1 is then defined as:

$$
(\mathcal{A} V, W)_{N}=A_{h}\left(\boldsymbol{v}_{h}, q_{h} ; \boldsymbol{w}_{h}, r_{h}\right) \quad \forall \boldsymbol{v}_{h}, \boldsymbol{w}_{h} \in V_{h}, \forall q_{h}, r_{h} \in Q_{h} .
$$

Since essential boundary conditions are applied for the velocity on the whole of $\partial \Omega$, the discrete pressure is only determined up to a constant, and so the matrix $\mathcal{A}$ has one zero eigenvalue. Throughout the remainder of this section, we therefore instead interpret $\mathcal{A}$ as $\mathcal{A}: \mathbb{R}^{N} / \operatorname{ker}(\mathcal{A}) \rightarrow$ $\operatorname{ker}(\mathcal{A})^{\perp}$, which is a bijective linear mapping by definition. The condition number of the matrix $\mathcal{A}$ is then defined by

$$
\kappa(\mathcal{A})=|\mathcal{A}|_{N}\left|\mathcal{A}^{-1}\right|_{N},
$$


with

$$
|\mathcal{A}|_{N}=\sup _{x \in \widehat{\mathbf{R}}^{N} \backslash \mathbf{0}} \frac{|\mathcal{A} x|_{N}}{|x|_{N}} .
$$

We now state some estimates that will be needed in the derivation of the estimate of the condition number. First, for a conforming, quasi-uniform mesh $\mathcal{T}$ with mesh size $h$ and a finite element space $\mathcal{V}_{h}$ defined on $\mathcal{T}$, it is well known that there are positive constants $c_{M}$ and $C_{M}$ only depending on the quasi-uniformness parameters and the polynomial order of $\mathcal{V}_{h}$ such that the following equivalence holds:

$$
c_{M} h^{d / 2}|V|_{N} \leqslant\left\|v_{h}\right\| \leqslant C_{M} h^{d / 2}|V|_{N} \quad \forall v_{h} \in \mathcal{V}_{h} .
$$

A second ingredient is an inverse estimate and a Poincaré inequality in the appropriate norms which we state in the following two lemmas.

Lemma 7.1 There is a constant $C_{I}$ such that

$$
\begin{aligned}
& \left\|\boldsymbol{v}_{h}\right\|_{*} \leqslant C_{I} h^{-1}\left\|\boldsymbol{v}_{h}\right\|_{\mathcal{T}_{1}^{*} \cup \mathcal{T}_{2}}, \quad \forall \boldsymbol{v}_{h} \in V_{h}, \\
& \left\|\left(\boldsymbol{v}_{h}, q_{h}\right)\right\|_{*} \leqslant C_{I} h^{-1}\left\|\left(\boldsymbol{v}_{h}, q_{h}\right)\right\|_{\mathcal{T}_{1}^{*} \cup \mathcal{T}_{2}} \quad \forall\left(\boldsymbol{v}_{h}, q_{h}\right) \in V_{h} \times Q_{h} .
\end{aligned}
$$

Proof By definition

$$
\left\|\mid \boldsymbol{v}_{h}\right\|_{*}^{2}=\left\|\nabla \boldsymbol{v}_{h}\right\|_{\mathcal{T}_{1}^{*} \cup \mathcal{T}_{2}}^{2}+\left\|\left\langle\partial_{\boldsymbol{n}} \boldsymbol{v}_{h}\right\rangle\right\|_{-1 / 2, h, \Gamma}^{2}+\left\|\left[\boldsymbol{v}_{h}\right]\right\|_{1 / 2, h, \Gamma}^{2}
$$

Applying the standard inverse inequality 4.10 on each mesh $\mathcal{T}_{1}^{*}$ and $\mathcal{T}_{2}$ separately, the first term is bounded by $C h^{-2}\left\|\boldsymbol{v}_{h}\right\|_{\mathcal{T}_{1}^{*} \cup \mathcal{T}_{2}}^{2}$. A similar bound can be derived for the second term by 4.11) and 4.10 . The estimate for the remaining term follows in the similar manner by a combination of 4.9 and again 4.10.

The second estimate 7.6 is an immediate consequence of the first and the fact that $1=C \leqslant$ $h^{-1} \operatorname{diam}(\Omega)$.

Lemma 7.2 There is a constant $C$ such that

$$
\|\boldsymbol{v}\|{\mathcal{\mathcal { T } _ { 1 }}}^{*} \cup \mathcal{T}_{2} \leqslant C_{P}\|\boldsymbol{v}\|_{*}, \quad \forall \boldsymbol{v} \in V_{h} .
$$

Proof By Proposition 4.1, we have

$$
\begin{aligned}
\|\boldsymbol{v}\|_{\mathcal{T}_{1}^{*} \cup \mathcal{T}_{2}}^{2} & =\|\boldsymbol{v}\|_{\mathcal{T}_{1}^{*}}^{2}+\|\boldsymbol{v}\|_{\mathcal{T}_{2}}^{2} \leqslant\|\boldsymbol{v}\|_{\Omega_{1}}^{2}+C\left(\|\nabla \boldsymbol{v}\|_{\mathcal{T}_{1}^{*}}^{2}+\|\boldsymbol{v}\|_{\Omega_{2}}^{2}\right) \\
& \leqslant C\left(\|\boldsymbol{v}\|_{*}^{2}+\|\boldsymbol{v}\|_{\Omega_{1} \cup \Omega_{2}}^{2}\right),
\end{aligned}
$$

for all $\boldsymbol{v} \in V_{h}$. Hence, it remains to derive a bound for a $\|\boldsymbol{v}\|_{\Omega_{1} \cup \Omega_{2}}$.

We use a duality argument to estimate $\|\boldsymbol{v}\|_{\Omega_{1} \cup \Omega_{2}}$. Let $\phi \in H^{2}(\Omega)$ be the solution of the dual problem $-\Delta \phi=\boldsymbol{v}$ with boundary conditions $\phi=0$ on $\partial \Omega$. Multiplying with $\boldsymbol{v}_{h}$ and integrating by parts we get by using the Cauchy-Schwarz inequality and the trace inequality 4.9 for $\phi$ :

$$
\begin{aligned}
\|\boldsymbol{v}\|_{\Omega_{1} \cup \Omega_{2}}^{2}= & \sum_{T \in \mathcal{T}_{1}}(\nabla \boldsymbol{v}, \nabla \boldsymbol{\phi})_{T \cap \Omega_{1}}+\sum_{T \in \mathcal{T}_{2}}(\nabla \boldsymbol{v}, \nabla \boldsymbol{\phi})_{T}-([\boldsymbol{v}], \boldsymbol{n} \cdot \nabla \boldsymbol{\phi})_{\Gamma} \\
\leqslant & \left(h^{-1}\|[\boldsymbol{v}]\|_{\Gamma}^{2}+\sum_{T \in \mathcal{T}_{1}^{*} \cup \mathcal{T}_{2}}\|\nabla \boldsymbol{v}\|_{T}^{2}\right)^{\frac{1}{2}} \\
& \times\left(\sum_{T \in \mathcal{T}_{1}^{*} \cup \mathcal{T}_{2}}\|\nabla \boldsymbol{\phi}\|_{T}^{2}+\sum_{T \in \mathcal{T}_{0, \Gamma}} C\left(\|\nabla \boldsymbol{\phi}\|_{T}^{2}+h^{2}|\nabla \boldsymbol{\phi}|_{1, T}^{2}\right)\right)^{\frac{1}{2}} \\
\leqslant & \|\boldsymbol{v}\|_{*}\|\boldsymbol{\phi}\|_{2, \Omega} .
\end{aligned}
$$

Finally, using standard elliptic regularity [6] $\|\phi\|_{2, \Omega} \leqslant\|\boldsymbol{v}\|_{\Omega}$ and division by $\|\boldsymbol{v}\|_{\Omega}$, the estimate follows. 
Theorem 7.1 The condition number of the stiffness matrix satisfies the estimate

$$
\kappa(\mathcal{A}) \leqslant C h^{-2} .
$$

Proof Recalling the definition of the condition number in 7.2 , the proof consists of deriving estimates of $|\mathcal{A}|_{N}$ and $\left|\mathcal{A}^{-1}\right|_{N}$. In the following, we use the following well-known equivalence for the Euclidean norm $|x|_{N}$ :

$$
|V|_{N}=\sup _{W \neq \mathbf{0}} \frac{(V, W)_{N}}{|W|_{N}}
$$

Estimate of $|\mathcal{A}|_{N}$. We have by the definition of $\mathcal{A}$

$$
\begin{aligned}
|\mathcal{A} V|_{N} & =\sup _{W \neq \mathbf{0}} \frac{(\mathcal{A} V, W)_{N}}{|W|_{N}} \\
& =\sup _{W \neq \mathbf{0}} \frac{A_{h}\left(\boldsymbol{v}_{h}, q_{h} ; \boldsymbol{w}_{h}, r_{h}\right)}{|W|_{N}} \\
& \leqslant C_{A} \sup _{W \neq \mathbf{0}} \frac{\left\|( \boldsymbol { v } _ { h } , q _ { h } ) \left|\left\|_{*} \cdot||\left(\boldsymbol{w}_{h}, r_{h}\right) \mid\right\|_{*}\right.\right.}{|W|_{N}} \\
& \leqslant C_{A} C_{I}^{2} \sup _{W \neq \mathbf{0}} \frac{h^{-1}\left\|\left(\boldsymbol{v}_{h}, q_{h}\right)\right\|_{\mathcal{T}_{1}^{*} \cup \mathcal{T}_{2}} \cdot h^{-1}\left\|\left(\boldsymbol{w}_{h}, r_{h}\right)\right\|_{\mathcal{T}_{1}^{*} \cup \mathcal{T}_{2}}}{|W|_{N}} \\
& \leqslant C_{A} C_{I}^{2} C_{M}^{2}|V|_{N} .
\end{aligned}
$$

Here, inequality (7.11) follows by the continuity of $A_{h}$ with respect to the norm $\|\cdot \cdot\| \|_{*}, 7.12$ follows by using the inverse estimate (7.6) twice, and finally (7.13) results by applying (7.4). Dividing by $|V|_{N}$ and taking the supremum over all $V \neq \mathbf{0}$, we conclude that

$$
|\mathcal{A}|_{N} \leqslant C_{A} C_{I}^{2} C_{M}^{2}
$$

Estimate of $\left|\mathcal{A}^{-1}\right|_{N}$. Starting with (7.4) and sequentially using the Poincaré inequality (7.7) (extended to the product space), the inf-sup stability (5.11) of $A_{h}$ and finally the Poincaré inequality again, we arrive at the following chain of estimates:

$$
\begin{aligned}
h^{d / 2}|V|_{N} & \leqslant c_{M}^{-1}\left\|\left(\boldsymbol{v}_{h}, q_{h}\right)\right\|_{\mathcal{T}_{1}^{*} \cup \mathcal{T}_{2}} \leqslant c_{M}^{-1} C_{P}\left\|\mid\left(_{h}, q_{h}\right)\right\| \|_{*} \\
& \leqslant c_{M}^{-1} C_{P} c_{A}^{-1} \frac{A\left(\boldsymbol{v}_{h}, q_{h} ; \boldsymbol{w}_{h}, r_{h}\right)}{\left\|\left(\boldsymbol{w}_{h}, r_{h}\right) \mid\right\|_{*}}=c_{M}^{-1} C_{P} c_{A}^{-1} \frac{(\mathcal{A} V, W)_{N}}{\left\|\left(\boldsymbol{w}_{h}, r_{h}\right)\right\|_{*}} \\
& \leqslant c_{M}^{-1} C_{P} c_{A}^{-1}|\mathcal{A} V|_{N} \frac{|W|_{N}}{\left\|\left(\boldsymbol{w}_{h}, r_{h}\right)\right\|_{*}} \\
& \leqslant c_{M}^{-2} C_{P} c_{A}^{-1}|\mathcal{A} V|_{N} h^{-d / 2} \frac{\left\|\left(\boldsymbol{w}_{h}, r_{h}\right)\right\|_{\mathcal{T}_{1}^{*} \cup \mathcal{T}_{2}}}{\left\|\left(\boldsymbol{w}_{h}, r_{h}\right) \mid\right\|_{*}} \\
& \leqslant c_{M}^{-2} C_{P}^{2} c_{A}^{-1}|\mathcal{A} V|_{N} h^{-d / 2}
\end{aligned}
$$

Setting $V=\mathcal{A}^{-1} W$ we have $W=\mathcal{A} V$ and the inequality now reads $\left|\mathcal{A}^{-1} W\right|_{N} \leqslant c_{M}^{-2} C_{P}^{2} c_{A}^{-1} h^{-d}|W|_{N}$ for all $W \neq 0$, or in other words

$$
\left|\mathcal{A}^{-1}\right|_{N} \leqslant c_{M}^{-2} C_{P}^{2} c_{A}^{-1} h^{-d} .
$$

Finally, combining (7.14 and 7.15) yields 7.8). 


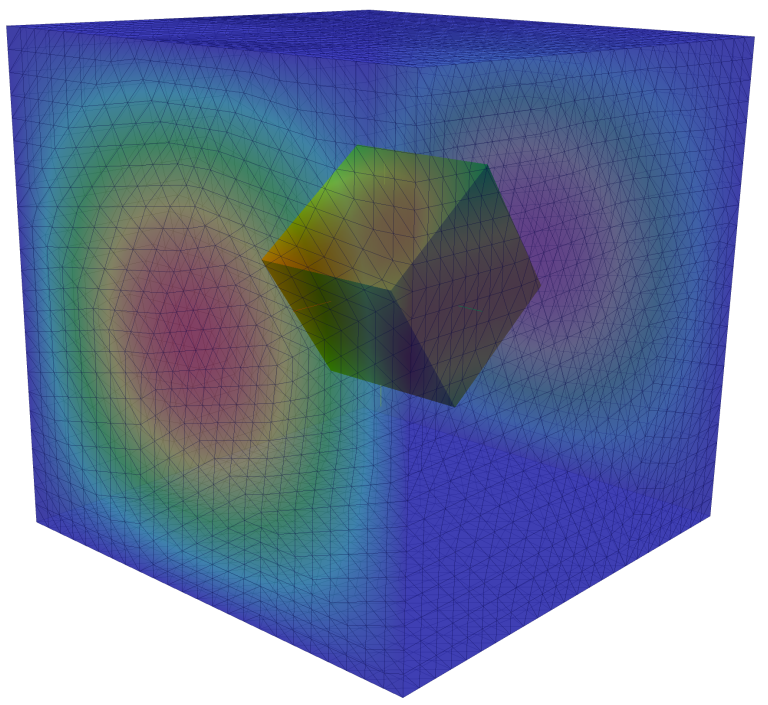

Fig. 8.1 Mesh configuration and magnitude of velocity approximation corresponding to this mesh resolution for the numerical convergence test.

\section{Numerical examples}

We conclude this paper with three numerical tests; all in three spatial dimensions. The numerical experiments were carried out using the DOLFIN-OLM library (http://launchpad.net/ dolfin-olm). We first corroborate the theoretical a priori error estimate with a convergence experiment. Second, we numerically examine how the location of the overlapping mesh in relation to the background mesh affects the condition number. Finally, we demonstrate how the method presented and the features provided by DOLFIN-OLM can be applied to the flow around an airfoil in a three dimensional channel. The experiments were performed with $V_{h}=V_{h}^{1}, Q_{h}=Q_{h}^{1}$ and $\beta=1$.

\subsection{Convergence test}

We let $\Omega=[0,1]^{3}$ and choose the overlapping domain $\Omega_{1}$ to be a rotation along the $y$-axis of the domain $\widetilde{\Omega}_{1}=[0.3331,0.6669]^{3}$ as illustrated in Figure 8.1 . To examine the convergence of the methods, we apply the method of manufactured solutions: let $\boldsymbol{u}(x, y, z)=(\sin (\pi y) \sin (\pi z), 0,0)$ and $p(x, y, z)=\cos (\pi x)+1$. The right-hand side $\boldsymbol{f}$ is defined accordingly so that $1.1 \mathrm{a}$ is satisfied, and the corresponding Dirichlet boundary conditions are strongly imposed on the entire boundary $\partial \Omega$. The numerical approximation corresponding to (3.7) is then computed on a sequence of overlapping uniform meshes $\left\{\left(\mathcal{T}_{0}^{N}, \mathcal{T}_{2}^{N}\right)\right\}_{N=0}^{5}$. The mesh size of the initial meshes $\mathcal{T}_{0}^{0}$ and $\mathcal{T}_{2}^{0}$ is $h_{\max } \approx 1 / 3$ and each subsequent pair $\left(\mathcal{T}_{0}^{N}, \mathcal{T}_{2}^{N}\right)$ is generated from the previous one by uniformly refining each mesh.

The stabilization parameters were set to $\gamma=10$ and $\delta=0.05$. To solve the linear system of equations, we apply a transpose-free quasi-minimal residual solver with an algebraic multigrid preconditioner, filtering out the constant pressure mode in the iterative solver. The solves converged to a tolerance of $10^{-8}$ in between 34 and 47 iterations.

The resulting errors for the sequence of refined meshes are given in Figure 8.2 Theorem 6.1 predicts first order convergence for both the $H^{1}$-norm of the velocity error and the $L^{2}$-norm of the pressure error. This is also (at least) observed in the numerical results, thus corroborating the 

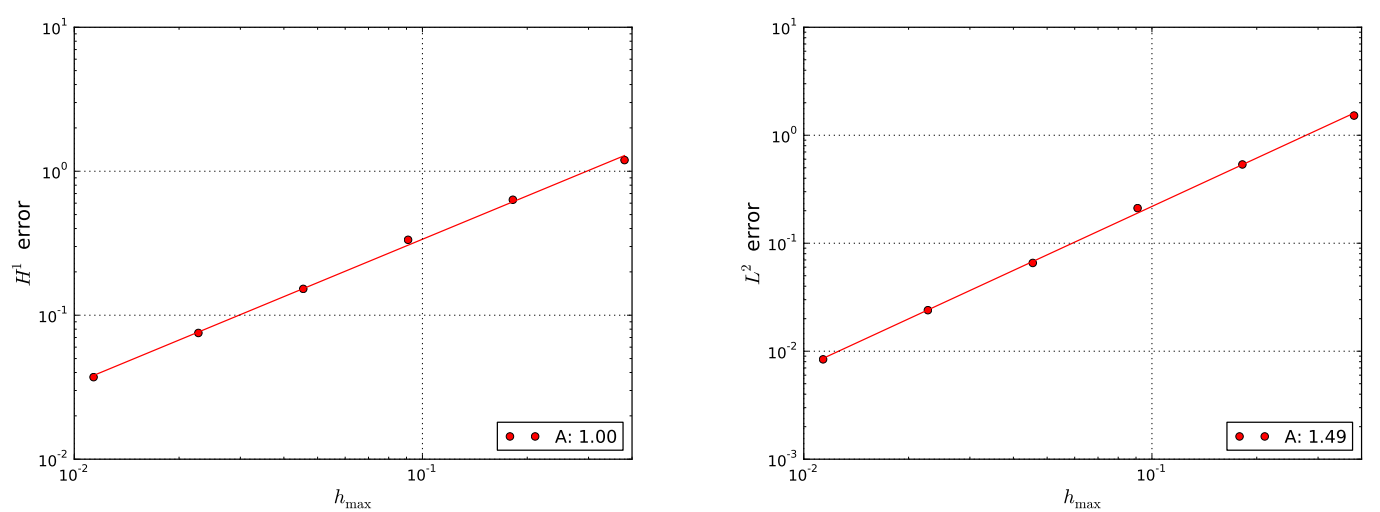

Fig. 8.2 Convergence of the stabilized Nitsche overlapping mesh method for $V_{h}^{1} \times Q_{h}^{1}$. The legend gives the fitted slope for each configuration. Top: $H^{1}$-error $\left\|\boldsymbol{u}-\boldsymbol{u}_{h}\right\|_{1, \mathcal{T}_{1}^{*} \cup \mathcal{T}_{2}}$ for the velocity versus maximal element diameter $h_{\max }$. Bottom: $L^{2}$-error $\left\|p-p_{h}\right\|_{\mathcal{T}_{1}^{*} \cup \mathcal{T}_{2}}$ for the pressure versus $h_{\max }$.

theoretical estimate. We additionally observe that the pressure approximation seems to converge at a higher order $(\approx 1.5)$ for the range of meshes investigated here.

\subsection{Condition number tests}

The next numerical example demonstrates that the condition number of the matrix $\mathcal{A}$ defined by (7.1) is bounded independently of the position of the overlapping mesh relative to the background mesh. This bound can be attributed to the term (3.4) defined on the overlap region: we also illustrate that the condition number seems unbounded if this term is not included.

Let $\Omega=[0,1]^{3}$ be tessellated by $N^{3}$ cubes, each cube divided into six tetrahedra. Define the overlapping domain by $\Omega_{2}=\Omega_{2}(l)=[l, 1-l]^{3}$ for a parameter $l \in(0,1)$, and tessellate $\Omega_{2}$ in the same manner as $\Omega$, but with $M^{3}$ cubes. We will consider two cases of mesh sizes: (i) $N=5, M=3$, and (ii) $N=10, M=6$. For both cases, $l$ approaching 0.2 from above corresponds to a limiting case: a few of the degrees of freedom in the overlapped mesh will only receive a contribution from the integrals defined over the overlap region while the cell and interface contributions vanish. Here, we therefore examine $l \in(0.2,0.21]$. For each $l$ and choice of $(N, M)$, we compute the condition number $\kappa$ of the corresponding matrix $\mathcal{A}$ as the ratio of the absolute values of the largest (in modulus) eigenvalue and the smallest (in modulus), nonzero, eigenvalue.

Let $\delta=0.05$ and $\gamma=10.0$ as before. The resulting condition numbers, scaled by the square mesh size of the overlapping mesh, are given in Table 8.1. We observe that for each $l$, the difference in the condition number between the two mesh sizes is small, as expected in view of Theorem 7.1 . Moreover, for both the case $N=5$ and $N=10$, the scaled condition numbers seem clearly bounded as $l \rightarrow 0.2$. In contrast, the scaled condition number grows significantly as $l \rightarrow 0.2$ when the overlap integrals $s_{h}$ are not included.

\begin{tabular}{l|rrrrr}
\hline Case & $l=0.21$ & 0.201 & 0.2001 & 0.20001 & 0.200001 \\
\hline$N=5(h \approx 0.33)$ & 1076 & 1207 & 1220 & 1222 & 1222 \\
$N=10(h \approx 0.17)$ & 955 & 1149 & 1170 & 1173 & 1174 \\
\hline$N=5$ without $s_{h}$ & 583 & 643 & 958 & 9715 & 110636 \\
\hline
\end{tabular}

Table 8.1 Scaled condition numbers $\kappa h^{2}$ where $h$ is the minimal cell diameter of the overlapping mesh. Each column to corresponds one overlapping domain $\Omega(l)$ for $l=0.21, l=0.201$ etc. The bottom row corresponds to the matrix induced by the form $A_{h}$ without the overlap integrals $s_{h}$. 


\subsection{Flow around an airfoil in a channel}

Finally, we illustrate that the method and technology developed here can be successfully applied to more realistic numerical simulations. In particular, we consider the flow around an airfoil in a channel.

As $\mathcal{T}_{2}$ we take a tetrahedral mesh approximation of a sphere surrounding an airfoil, discretizing the boundary layer around the airfoil with a higher mesh resolution than the remainder of the domain. As $\mathcal{T}_{0}$ we take a tessellation of $[-3,3]^{3} \backslash O$ where the interior domain $O$ is contained in the convex hull of $\mathcal{T}_{2}$. (In total, $\mathcal{T}_{0} \cup \mathcal{T}_{2}$ is a mesh of a $[-3,3]^{3}$ box with an airfoil removed.)

The stabilization parameters and choice of finite element spaces are as before: $V_{h}^{1} \times Q_{h}^{1}, \beta=1$, $\delta=0.05$ and $\gamma=10$. We enforce a parabolic velocity profile at the inflow boundary, no slip conditions for the velocity on the outer walls and the airfoil boundary, and stress-free boundary conditions at the outflow boundary and take $\boldsymbol{f}=\mathbf{0}$.

The flow patterns around the airfoil can now be studied for instance for different angles of attack by rotating $\mathcal{T}_{2}$ while keeping $\mathcal{T}_{0}$ fixed. The velocity and pressure approximations for a series of mesh pairs, in which $\mathcal{T}_{2}$ was first rotated around the $z$-axis with angle $\theta$ and then around the $y$-axis with the same angle, are visualized in Figures 8.3 and 8.4 . We especially note the smooth transition of the solution from $\mathcal{T}_{0}$ to $\mathcal{T}_{2}$; the interface is not visible.

\section{Conclusions}

We have presented and analyzed a general class of stabilized finite element methods for the Stokes problem posed on overlapping, non-matching meshes. The interface conditions are enforced using Nitsche's method, yielding a provably optimally convergent method. In addition, a least-squares term on the overlap region is included to control the condition number of the stiffness matrix.

The theoretical results have been verified numerically for a test problem consisting of Stokes flow through a channel described by a sphere superimposed on a tetrahedral mesh of the unit cube. We have further verified that the condition number of the stiffness matrix remains bounded, independently of the location of the overlapping mesh relative to the background mesh. Finally, we have demonstrated the applicability of the proposed method and our implementation to the simulation of Stokes flow around a three-dimensional airfoil with a fitted mesh superimposed on a non-matching background mesh.

A natural extension of the work presented in this paper is to the simulation of fluid-structure interaction problems where a fluid mesh of the surroundings of an elastic body is superimposed on a background fluid mesh as in Figure 1.1. The fluid-structure interaction may then be handled via a standard arbitrary Lagrangian-Eulerian (ALE) approach on the overlapping mesh, while the Nitsche overlapping mesh method analyzed in this paper is used to enforce the interface conditions across the fluid-fluid boundary. We explore this technique further in ongoing work.

While all software used in this work is available as free/open-source, additional work is required to create interfaces and documentation that make the software useful to a general audience. This issue will be addressed in the near future, with the goal to provide an easy-to-use programming environment for overlapping mesh methods as part of the FEniCS software suite.

Acknowledgements This work is supported by an Outstanding Young Investigator grant from the Research Council of Norway, NFR 180450. This work is also supported by a Center of Excellence grant from the Research Council of Norway to the Center for Biomedical Computing at Simula Research Laboratory. 

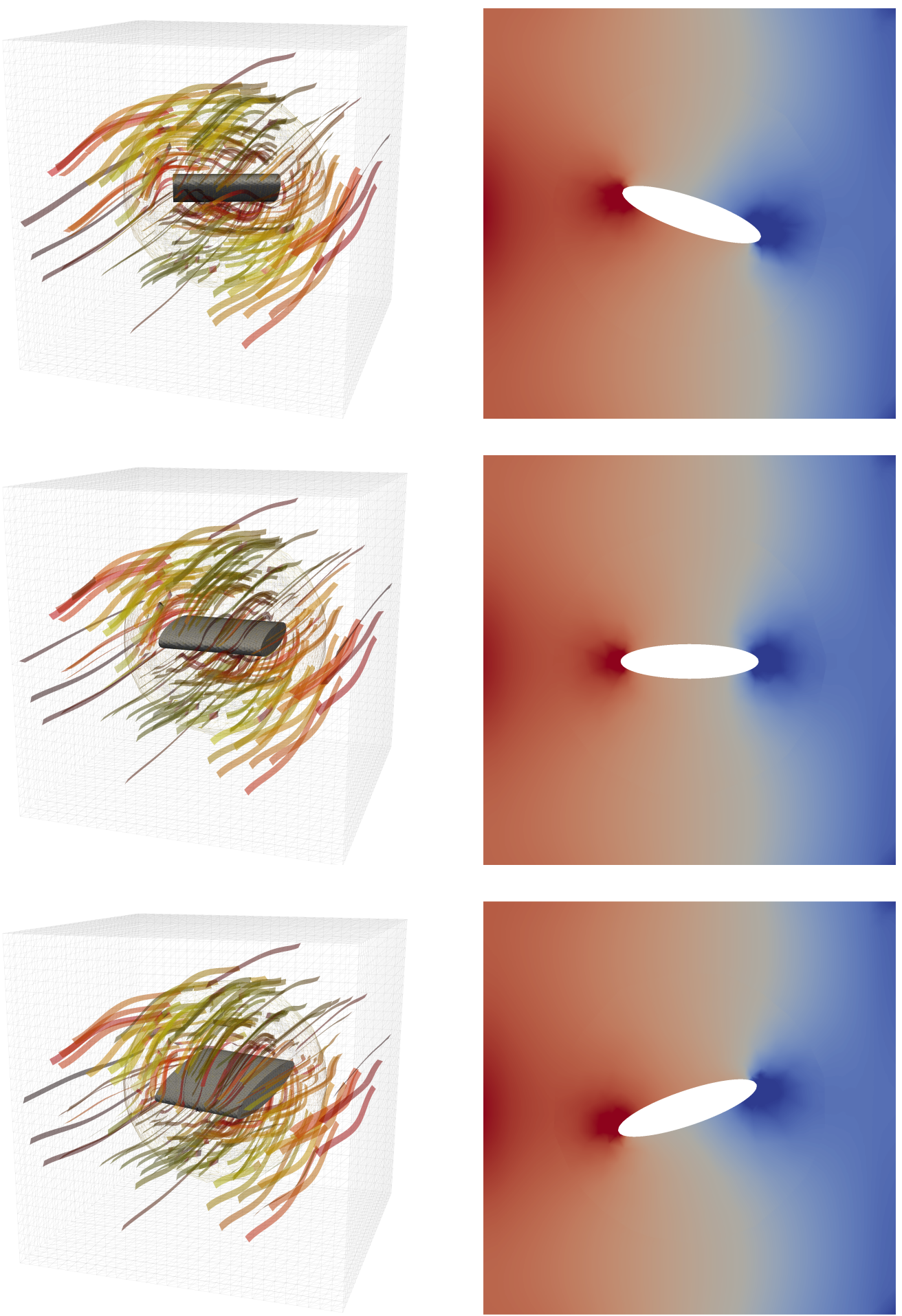

Fig. 8.3 Velocity streamlines (left) and pressures in the plane defined by the $z$-axis (right) for different angles of attack $\theta$ : from top to bottom: $\theta=20^{\circ}, 0^{\circ},-20^{\circ}$. 

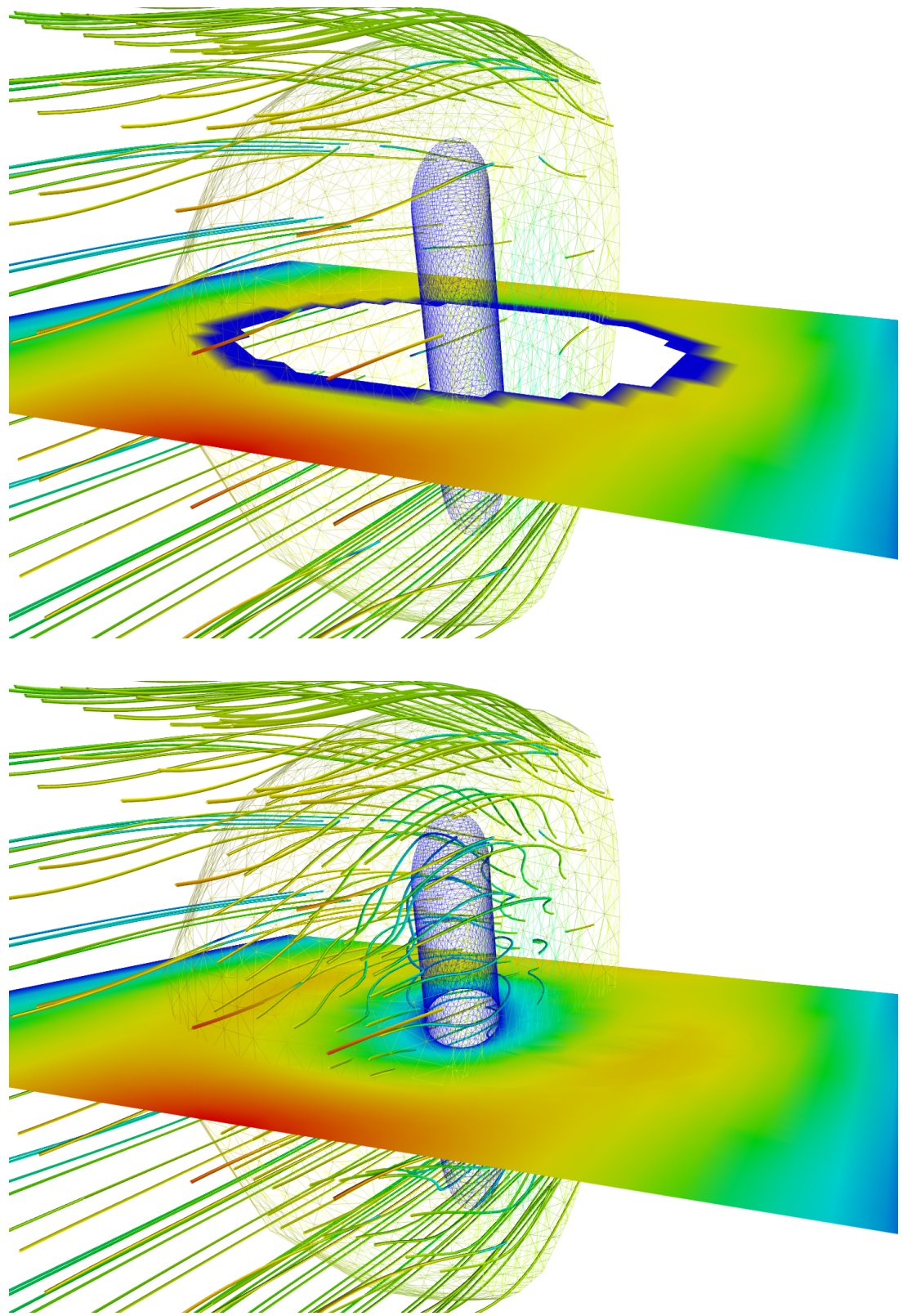

Fig. 8.4 Velocity streamlines and magnitude in a cross-section for angle of attack $\theta=-20^{\circ}$. Top: The solution on the background mesh $\mathcal{T}_{1}^{*}$. It can clearly be seen that the streamlines stop close to the interface zone. Bottom: The solution on the overlapping mesh $\mathcal{T}_{2}$ superimposed on the solution on the background mesh $\mathcal{T}_{1}^{*}$, indicating a smooth transition of the solution from $\mathcal{T}_{1}^{*}$ to $\mathcal{T}_{2}$. 


\section{References}

1. Martin S. Alnæs. UFL: a Finite Element Form Language, chapter 17. Springer, 2012.

2. Martin S. Alnæs, Anders Logg, Kent-Andre Mardal, Ola Skavhaug, and Hans Petter Langtangen. Unified framework for finite element assembly. Int. J. Comput. Sci. Eng., 4(4):231-244, 2009.

3. D. N. Arnold, F. Brezzi, B. Cockburn, and L. D. Marini. Unified analysis of discontinuous Galerkin methods for elliptic problems. SIAM J. Num. Anal., 39:1749-1779, 2002.

4. Teri Barth, Pavel Bochev, Max Gunzburger, and John Shadid. A Taxonomy of Consistently Stabilized Finite Element Methods for the Stokes Problem. SIAM J. Num. Anal., 25(5):1585, 2004.

5. Roland Becker, Erik Burman, and Peter Hansbo. A Nitsche extended finite element method for incompressible elasticity with discontinuous modulus of elasticity. Comput. Methods Appl. Mech. Engrg., 198(41-44):3352-3360, 2009.

6. Susanne C. Brenner and L. Ridgway Scott. The mathematical theory of finite element methods, volume 15 of Texts in Applied Mathematics. Springer, New York, third edition, 2008.

7. D. Day and P. Bochev. Analysis and computation of a least-squares method for consistent mesh tying. J. Comput. Appl. Math., 218(1):21-33, August 2008.

8. J. Douglas and J. Wang. An absolutely stabilized finite element method for the Stokes problem. Math. Comp, 52(186):495--508, 1989.

9. A. Ern and J.L. Guermond. Evaluation of the condition number in linear systems arising in finite element approximations. ESAIM, Math. Model. Num. Anal., 40(1):29-48, 2006.

10. L.P. Franca, T.J.R. Hughes, and R. Stenberg. Stabilized finite element methods for the Stokes problem. In M.D. Gunzburger and R. A. Nicolaides, editors, Incompressible Computational Fluid Dynamics. Cambridge University Press, 1993.

11. V. Girault, B. Rivière, and M. F. Wheeler. A discontinuous Galerkin method with nonoverlapping domain decomposition for the Stokes and Navier-Stokes problems. Math. Comp., 74 (249):53-84, 2005.

12. A. Hansbo and P. Hansbo. An unfitted finite element method, based on Nitsche's method, for elliptic interface problems. Comput. Methods Appl. Mech. Engrg., 191(47-48):5537-5552, 2002.

13. A. Hansbo, P. Hansbo, and Mats G. Larson. A Finite Element Method on Composite Grids based on Nitsche's Method. ESAIM, Math. Model. Num. Anal., 37(3):495-514, 2003.

14. P. Hansbo. Nitsche's method for interface problems in computational mechanics. GAMM-Mitt, 28(2):183-206, 2005.

15. P. Hansbo and J. Hermansson. Nitsche's method for coupling non-matching meshes in fluidstructure vibration problems. Comput. Mech., 32(1-2):134-139, 2003.

16. Thomas J. R. Hughes, Leopoldo P. Franca, and Marc Balestra. A new finite element formulation for computational fluid dynamics. V. Circumventing the Babuška-Brezzi condition: a stable Petrov-Galerkin formulation of the Stokes problem accommodating equal-order interpolations. Comput. Methods Appl. Mech. Engrg., 59(1):85-99, 1986.

17. Robert C. Kirby and Anders Logg. A Compiler for Variational Forms. ACM Trans. Math. Softw., 32(3):417-444, 2006.

18. Anders Logg. Automating the finite element method. Arch. Comput. Methods Eng., 14(2): 93-138, 2007.

19. Anders Logg and Garth N. Wells. DOLFIN: Automated finite element computing. ACM Trans. Math. Softw., 37(2), 2010.

20. Anders Logg, Kent-Andre Mardal, Garth N. Wells, et al. Automated Solution of Differential Equations by the Finite Element Method. Springer, 2012.

21. Anders Logg, Kristian B. Ølgaard, Marie E. Rognes, and Garth N. Wells. FFC: the FEniCS Form Compiler, chapter 11. Springer, 2012.

22. A. Massing, Mats G. Larson, and A. Logg. Efficient implementation of finite element methods on non-matching and overlapping meshes in 3D. submitted, 2012. 
23. A. Massing, Mats G. Larson, A. Logg, and Marie E. Rognes. A stabilized Nitsche fictitious domain method for the Stokes problem. submitted, 2012.

24. J. Nitsche. Über ein Variationsprinzip zur Lösung von Dirichlet-Problemen bei Verwendung von Teilräumen, die keinen Randbedingungen unterworfen sind. Abhandlungen aus dem Mathematischen Seminar der Universität Hamburg, 36(1):9-15, July 1971.

25. Alfio Quarteroni. Numerical Models for Differential Problems. Modeling, Simulation and Applications. Springer-Verlag, 2009.

26. R. Scott and S. Zhang. Finite element interpolation of nonsmooth functions satisfying boundary conditions. Math. Comp., 54(190):483-493, 1990.

27. E. Stein. Singular Integrals and Differentiability Properties of Functions. Princeton University Press, 1970.

28. R. Verfürth. A posteriori error estimation and adaptive mesh-refinement techniques. In Proceedings of the fifth international conference on Computational and applied mathematics table of contents, pages 67-83. Elsevier Science Publishers BV Amsterdam, The Netherlands, The Netherlands, 1994. 